ARTICLE

\title{
Coupling of 5S RNP rotation with maturation of functional centers during large ribosomal subunit assembly
}

Jelena Micic ${ }^{1,7}$, Yu Li (iD) ${ }^{2,3,7}$, Shan Wu ${ }^{2,4}$, Daniel Wilson', Beril Tutuncuoglu (iD ${ }^{5}$, Ning Gao (i) ${ }^{6 凶} \&$ John L. Woolford Jr. (10 ${ }^{1 凶}$

The protein composition and structure of assembling $60 \mathrm{~S}$ ribosomal subunits undergo numerous changes as pre-ribosomes transition from the nucleolus to the nucleoplasm. This includes stable anchoring of the Rpf2 subcomplex containing 5S rRNA, rpL5, rpL11, Rpf2 and Rrs1, which initially docks onto the flexible domain $\mathrm{V}$ of rRNA at earlier stages of assembly. In this work, we tested the function of the C-terminal domain (CTD) of Rpf2 during these anchoring steps, by truncating this extension and assaying effects on middle stages of subunit maturation. The rpf2 255-344 mutation affects proper folding of rRNA helices H68-70 during anchoring of the Rpf2 subcomplex. In addition, several assembly factors (AFs) are absent from pre-ribosomes or in altered conformations. Consequently, major remodeling events fail to occur: rotation of the 5S RNP, maturation of the peptidyl transferase center (PTC) and the nascent polypeptide exit tunnel (NPET), and export of assembling subunits to the cytoplasm.

\footnotetext{
${ }^{1}$ Department of Biological Sciences, Carnegie Mellon University, Pittsburgh, PA, USA. ${ }^{2}$ State Key Laboratory of Membrane Biology, School of Life Science, Tsinghua University, Beijing, China. ${ }^{3}$ Peking University-Tsinghua University-National Institute of Biological Sciences Joint Graduate Program, Beijing, China. ${ }^{4}$ State Key Laboratory of Biocatalysis and Enzyme Engineering, Hubei Collaborative Innovation Center for Green Transformation of Bio-Resources, School of Life Sciences, Hubei University, Wuhan, China. ${ }^{5}$ Department of Cellular and Molecular Pharmacology, University of California, San Francisco, CA, USA. ${ }^{6}$ State Key Laboratory of Membrane Biology, Peking-Tsinghua Center for Life Sciences, School of Life Sciences, Peking University, Beijing, China. ${ }^{7}$ These

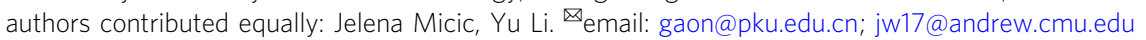


$\mathrm{R}$ ibosomes translate the genetic code in mRNA and catalyze the synthesis of proteins in all cells in nature. They are comprised of two subunits. In yeast, the large (60S) subunit includes 3 ribosomal RNAs (rRNAs) (25S, 5.8S, and 5S) and 46 ribosomal proteins (r-proteins), whereas the small (40S) subunit contains 1 rRNA (18S) and 33 r-proteins. Ribosomal subunit assembly involves transcription, modification, processing and folding of rRNA, and binding of r-proteins ${ }^{1-6}$. More than 200 assembly factors (AFs) facilitate accurate and efficient ribosome biogenesis, enabling proper growth and proliferation of cells ${ }^{1,6}$.

In $60 \mathrm{~S}$ ribosomal subunits, $25 \mathrm{~S}$ rRNA is divided into six phylogenetically conserved secondary structure domains. During assembly, folding and compaction of rRNA is coupled with stable incorporation of $\mathrm{r}$-proteins, forming complex ribonucleoprotein (RNP) particles ${ }^{7}$. Assembly of $60 \mathrm{~S}$ subunits is hierarchical; rRNA domains that form the convex solvent side of the subunit assemble first, followed by those that comprise the nascent polypeptide exit tunnel (NPET), and finally the central protuberance $(\mathrm{CP})$ and the subunit interface containing the peptidyl transferase center $(\mathrm{PTC})^{8-11}$.

Three out of four rRNAs (18S, 25S, and 5.8S) are transcribed by RNA polymerase I and processed from a single rRNA precursor (35S pre-rRNA), whereas 5S rRNA is transcribed separately by RNA polymerase III and is processed independently (Supplementary Fig. 1a) ${ }^{12,13}$. The 5S rRNA forms the 5S RNP complex with two r-proteins, rpL5 and rpL11, in the cytoplasm. This complex is imported to the nucleolus by the transport adaptor Syo $1^{14,15}$ and binds to the portion of $25 \mathrm{~S}$ rRNA domain $\mathrm{V}$ that becomes part of the $\mathrm{CP}$ in mature subunits ${ }^{7}$.

In metazoa, stable association of the 5S RNP with assembling $60 \mathrm{~S}$ subunits is coordinated with cell growth and proliferation. When ribosomal assembly fails, the free 5S RNP binds to MDM2, an inhibitor of the tumor suppressor p53, and sequesters it in the nucleolus. This activates p53, cell cycle arrest, and eventually apoptosis. In addition, rpL11 inhibits other potential oncogenic properties of MDM2 $2^{16-18}$.

The $5 S$ RNP is a part of the Rpf2 subcomplex, together with a heterodimer of AFs Rpf2 and Rrs1 ${ }^{19-23}$. Rpf2 and Rrs1 are necessary for initial docking of the 5S RNP with the assembling subunit, but the timing of their entry remains unclear ${ }^{20}$. Rpf2 and Rrs1 may assemble into the subunit before the 5S RNP or they may all associate at the same time 22,23 . All of them copurify with early nucleolar assembly intermediates ${ }^{20,24,25}$. However, they cannot be visualized on particles by cryo-electron microscopy (cryo-EM) until the formation of Nog2 State 1/State F/Arx1 particles during late nucleolar stages (middle stages) of $60 \mathrm{~S}$ subunit assembly (Supplementary Figs. $1 \mathrm{~b}$ and 2a) 9,26,27. This transition from a flexible/invisible state to a stable/visible state is designated as "anchoring". Anchoring includes stable positioning of the Rpf2 subcomplex to the region that will become the CP and the specific rRNA rearrangements initiated by this positioning. Depletions of the Rpf2 subcomplex constituents do not affect biogenesis of $60 \mathrm{~S}$ subunits until this anchoring occurs ${ }^{20,28}$. Thus, the Rpf2 subcomplex and rRNA domain $\mathrm{V}$ (helices H80, H82-88) with which it first associates are initially docked in a flexible state and do not influence assembly of nascent subunits until they become stably anchored within Nog2 particles.

Strikingly, when the Rpf2 subcomplex anchors onto assembling pre-60S subunits, the $5 \mathrm{~S} \mathrm{RNP}$ is in a $\sim 180^{\circ}$-rotated position compared with that in mature $60 \mathrm{~S}$ subunits ${ }^{26}$. The adjacent rRNA helices (H80, H82-H88), as well as H38, H69, and H70, also display drastic conformational differences compared with mature subunits $^{26}$. In order for 5S RNP rotation to occur, Rpf2 and Rrs1 must exit assembling subunits. Their exit appears to be initiated by binding of Sda1 in close proximity to the Rpf2-Rrs1 dimer and H68-H69. The exact mechanism of this process is still unclear. Sda1 then recruits the Rix1 subcomplex (Ipi1/Rix1/Ipi3) and the $\mathrm{AAA}+\mathrm{ATPase}$ Rea1. Their initial binding is thought to destabilize interactions between the 5S RNP, Rpf2-Rrs1, and Rsa4 to allow rotation of $\mathrm{H} 38$ and $5 \mathrm{~S}$ RNP. These structural rearrangements may activate Rea1, which removes Rsa4 and itself using ATP hydrolysis. Finally, Nog2 must exit using its own GTPase activity to allow the nuclear export factor $\mathrm{Nmd} 3$ to replace it, thus enabling export competency (Supplementary Fig. 1b) 14,19,26,27,29-32.

Importantly, during middle stages of $60 \mathrm{~S}$ subunit assembly, other significant structural rearrangements also occur. They include the following: construction of future functional centers (PTC and NPET), removal of the rRNA spacer ITS2, and anchoring of rRNA domains IV and $\mathrm{V}$ to the main body of the subunit. Whether and how these significant rearrangements are coordinated and interconnected is unclear.

Intriguingly, the partially disordered C-terminal domain (CTD) of Rpf 2 is positioned between the GTPase domain of Nog2 and helices $\mathrm{H} 69-\mathrm{H} 71^{27}$. Here, we have truncated this extension to investigate its function in middle stages of assembly, in particular its potential involvement in stable anchoring of the Rpf2 subcomplex to the nascent pre-60S particle. Our results indicate that this long extension of Rpf 2 does not appear to be necessary for docking of the Rpf2 subcomplex to rRNA domain $\mathrm{V}$ during the early stages of assembly, although a small effect cannot be ruled out. Instead, it is necessary, directly or through Nog2, for proper folding of H68-70 during anchoring. As a result, improper conformations of these helices affect binding of AFs necessary for rotation of the 5S RNP, maturation of the NPET, and export of nascent $60 \mathrm{~S}$ subunits to the cytoplasm. H68-H70 are also involved in accurate formation of the PTC during subunit biogenesis, are part of inter-subunit bridges with the $40 \mathrm{~S}$ ribosomal subunit, and are crucial for efficient protein synthesis ${ }^{33-36}$. In summary, conformational immaturity of these rRNA helices in the rpf2 $2255-344$ mutant strain blocks 60 S ribosomal subunit assembly during late nucleolar steps.

\section{Results}

The CTD of Rpf 2 is involved in $60 \mathrm{~S}$ subunit assembly. To understand the role of the Rpf2 subcomplex during middle stages of subunit assembly, we made specific mutations in the AF Rpf2. We took advantage of a high-resolution cryo-EM structure of the Nog2 particle containing the 5S RNP, Rpf2, and Rrs1 (Supplementary Fig. 2a) ${ }^{27}$. Rpf2 consists of a globular domain that interacts extensively with $\mathrm{Rrs}^{21-23}$ and an extended CTD (amino acids 255-344) (Supplementary Fig. 2b-d). Although amino acids 255-300 in the CTD of Rpf2 are highly conserved, amino acids 301-344 are not (Supplementary Fig. 3). The latter are predicted to be disordered and are not visible by cryo-EM in Nog2 particles, presumably due to their flexibility. The presence of an epitope tag at the $\mathrm{C}$ terminus of $\mathrm{Rpf} 2(R P F 2-T A P)$ affects cell growth and production of $60 \mathrm{~S}$ ribosomal subunits (Supplementary Fig. 4a), suggesting the importance of the CTD of Rpf 2 in maturation of pre-60S ribosomes. The CTD of Rpf2 is positioned between the GTPase domain of Nog2 and rRNA helices H68-H71; thus, it may contribute to maturation of these helices (Supplementary Fig. 2b) ${ }^{27}$. Therefore, we deleted the entire CTD (amino acids 255-344) or only the disordered portion of the CTD (amino acids 310-344). We also created two clusters of mutations in conserved sequences of the CTD, which would potentially disrupt interactions between Rpf2 and Nog2, and Rpf2 and H68-71. The rpf2-1 allele (cluster 1) contains two substitution mutations (I267A and M268A), whereas the rpf2-2 allele (cluster 2) contains six mutations (I275A, M277A, G278A, Q280A, L282A, and L285A) (Supplementary Fig. 2c). 
a

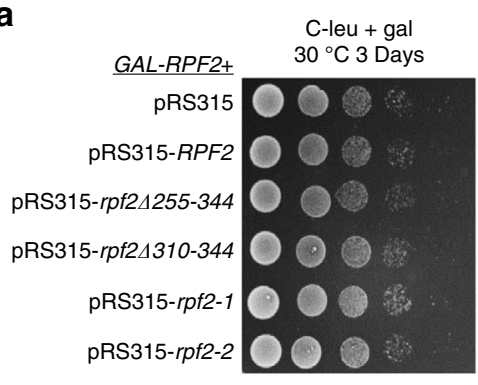

b

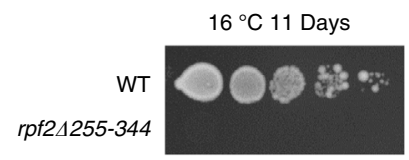

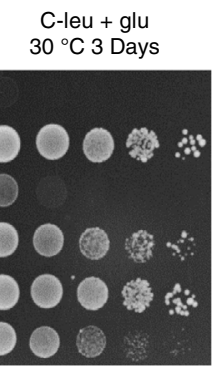

$25^{\circ} \mathrm{C} 3$ Days

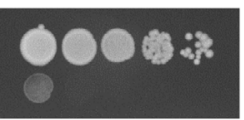

C-leu + glu

$16^{\circ} \mathrm{C} 7$ Days

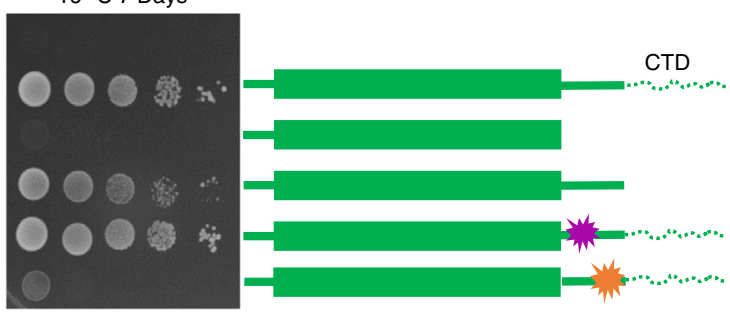

$30^{\circ} \mathrm{C} 3$ Days

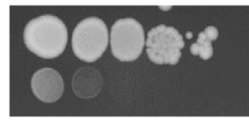

$37^{\circ} \mathrm{C} 3$ Days

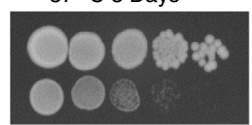

Fig. 1 The CTD of Rpf2 is essential for cell growth at $16^{\circ} \mathbf{C}$. a Left: growth of yeast strains containing truncated or mutated Rpf 2 protein on solid medium. Serial dilutions (1:10 to $1: 10,000$ ) were spotted onto selective solid medium containing either galactose or glucose and incubated at $30{ }^{\circ} \mathrm{C}$ and $16^{\circ} \mathrm{C}$. Right: schematic representation of truncations and mutations introduced into the CTD of Rpf2. Amino acids 301-344 are represented by dashed lines. b Growth of yeast strain containing the genomic allele of rpf2 $2555-344$ and its wild-type control. Serial dilutions $(1: 10$ to $1: 10,000)$ were spotted onto solid medium and incubated at $16,25,30$, and $37^{\circ} \mathrm{C}$. Source data are provided as a Source Data file.

To assay the effects of these mutations, we expressed mutant proteins from plasmids in a strain conditional for expression of endogenous Rpf2 protein (GAL-RPF2).

We assayed growth of the mutant strains on galactose- (where both wild-type RPF2 and mutant $r p f 2$ are expressed) or glucosecontaining solid media (where only mutant $r p f 2$ is expressed) at different temperatures. Removal of the entire CTD ( $r p f 2 \Delta 255$ 344) had a strong growth defect at all temperatures and was lethal at $16^{\circ} \mathrm{C}$. Truncation of the disordered portion of the CTD (rpf2 $\Delta 310-344)$ had a very slight growth defect at $16^{\circ} \mathrm{C}$. Thus, the conserved portion of the CTD appears to play a more important role in $60 \mathrm{~S}$ subunit assembly than the disordered domain. The rpf2-1 mutation did not affect growth. The $r p f 2-2$ mutant had a slight growth defect at $30^{\circ} \mathrm{C}$ but growth was affected strongly at $16^{\circ} \mathrm{C}$, similar to the $r p f 2 \Delta 255-344$ mutant (Fig. 1a).

Based on these growth defects, we focused on the rpf2 $\Delta 255-344$ mutant. To determine whether the truncated Rpf2 protein produced by this mutant was stable, we tagged both Rpf 2 and rpf2 $\Delta 255-344$ proteins with double Myc epitopes at their $\mathrm{N}$ termini. We tested the expression and stability of these proteins, as well as two other protein constituents of the Rpf2 subcomplex (rpL5 and rpL11), by western blotting of whole-cell lysates (Supplementary Fig. 4b). All assayed proteins were stably expressed.

To test the ability of the mutant Myc-rpf2 $\Delta 255-344$ protein to enter pre-ribosomal subunits, we performed sucrose gradient fractionation. As expected, the levels of free $60 \mathrm{~S}$ subunits were significantly lower relative to $40 \mathrm{~S}$ subunits in the $r p f 2 \Delta 255-344$ strain, compared with the wild-type strain, indicating a defect in $60 \mathrm{~S}$ subunit assembly. In addition, polysomes (translating ribosomes) were decreased in the mutant strain (Supplementary Fig. 4c). Importantly, levels of the truncated rpf2 protein sedimenting with pre-60S particles compared with levels at the top of the gradient (free proteins and small complexes) were comparable to the wild-type protein (Supplementary Fig. 4d). Together, these results indicate that the truncated Rpf 2 is stable and can enter pre-ribosomal particles at wild-type levels.

As the rpf2 $\Delta 255-344$ mutation is conditionally lethal (cold sensitive), we created a strain in which the genomic wild-type $R P F 2$ allele was replaced with the rpf2 255 -344 mutant allele. Yeast containing this mutant allele in the genome grew slowly at all temperatures and were inviable at $16^{\circ} \mathrm{C}$ (Fig. 1b). Subsequent experiments were performed using yeast containing the genomic $r p f 2 \Delta 255-344$ mutant strain and yeast expressing the plasmidborne mutant allele, with identical results.

To examine at what step of the $60 \mathrm{~S}$ subunit assembly pathway the $r p f 2 \Delta 255-344$ mutant is blocked, we assayed levels of prerRNAs by northern blotting and primer extension assays. The mutant strain accumulated $27 \mathrm{SB}$ and $7 \mathrm{~S}$ pre-rRNAs, indicating a defect in late nucleolar/nucleoplasmic (middle) stages of $60 \mathrm{~S}$ subunit assembly, during the lifetime of Nog2 particles (Supplementary Figs. 1a and $4 \mathrm{e}-\mathrm{g}$ ).

To confirm that $60 \mathrm{~S}$ subunit assembly in the $r p f 2 \Delta 255-344$ mutant is blocked prior to subunit export from the nucleus to the cytoplasm, we assayed the localization of nascent pre-60S ribosomes, using the rpL25-eGFP reporter. In wild-type RPF2 cells, rpL25eGFP was most abundantly present in the cytoplasm as part of translating ribosomes (Supplementary Fig. 5a, top panels). In the rpf2 $\Delta 255$-344 mutant, rpL25eGFP accumulated in the nucleus, with some nucleolar, but mostly nucleoplasmic localization (Supplementary Fig. 5a, bottom panels). This result indicates that middle stages of assembly are blocked, resulting in failure to export pre-60S particles to the cytoplasm.

Truncation of the CTD of Rpf2 affects levels of specific AFs. To uncover changes in pre-ribosomal protein composition in the $r p f 2 \Delta 255-344$ mutant, we affinity-purified pre-ribosomal particles from the middle stages of $60 \mathrm{~S}$ subunit maturation and examined their protein constituents by SDS-polyacrylamide gel electrophoresis (PAGE), western blotting, and semi-quantitative mass spectrometry (iTRAQ) (Fig. 2 and Supplementary Fig. 5c). As Nog2 is present in pre-ribosomes during the middle stages of $60 \mathrm{~S}$ subunit assembly (Supplementary Fig. 1b) and it accumulates in Nop7 particles purified from the rpf2 $\Delta 255-344$ mutant strain (indicating a block in assembly during the lifetime of Nog2 in pre-ribosomes) (Supplementary Fig. 5b), we used epitope-tagged Nog2 as a bait for these purifications.

Levels of AFs Sda1, the Rix1 subcomplex (Ipi1, Rix1/Ipi2, and Ipi3), and Rea1 decreased significantly in the Nog2 particles lacking the CTD of Rpf2 (Fig. 2). These AFs have been previously shown to be necessary for rotation of $5 \mathrm{~S} \mathrm{RNP}^{19,37}$. Rsa4, which requires Rea1 for its release from preribosomes, accumulated slightly. In addition, levels of nuclear export factors Arx1, Bud20, Ecm1, as well as the Arx1-associated protein Alb1, were 
a

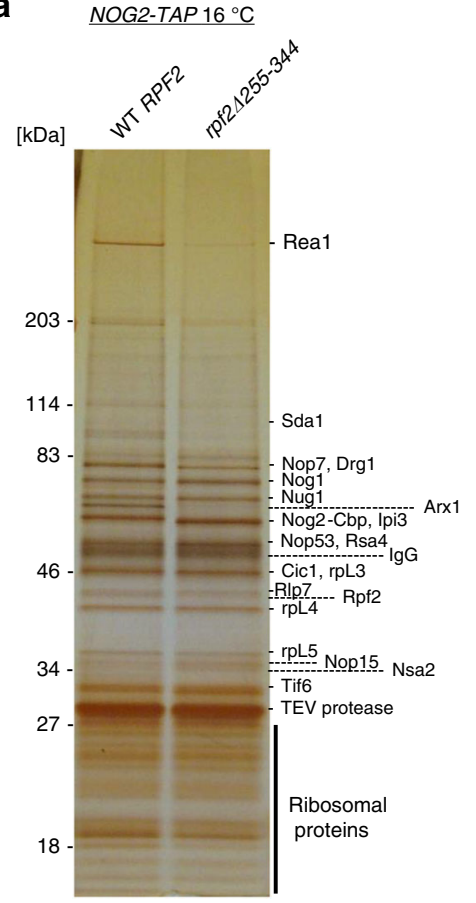

b

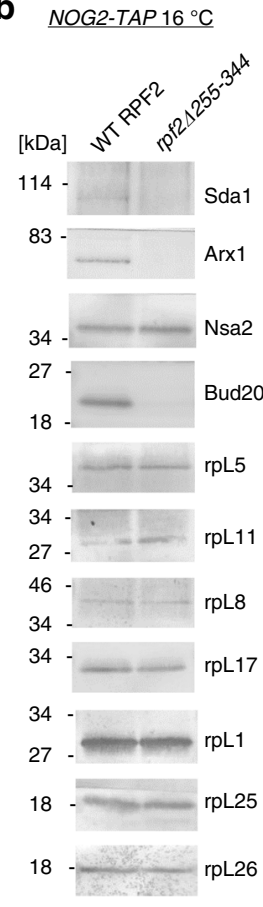

C

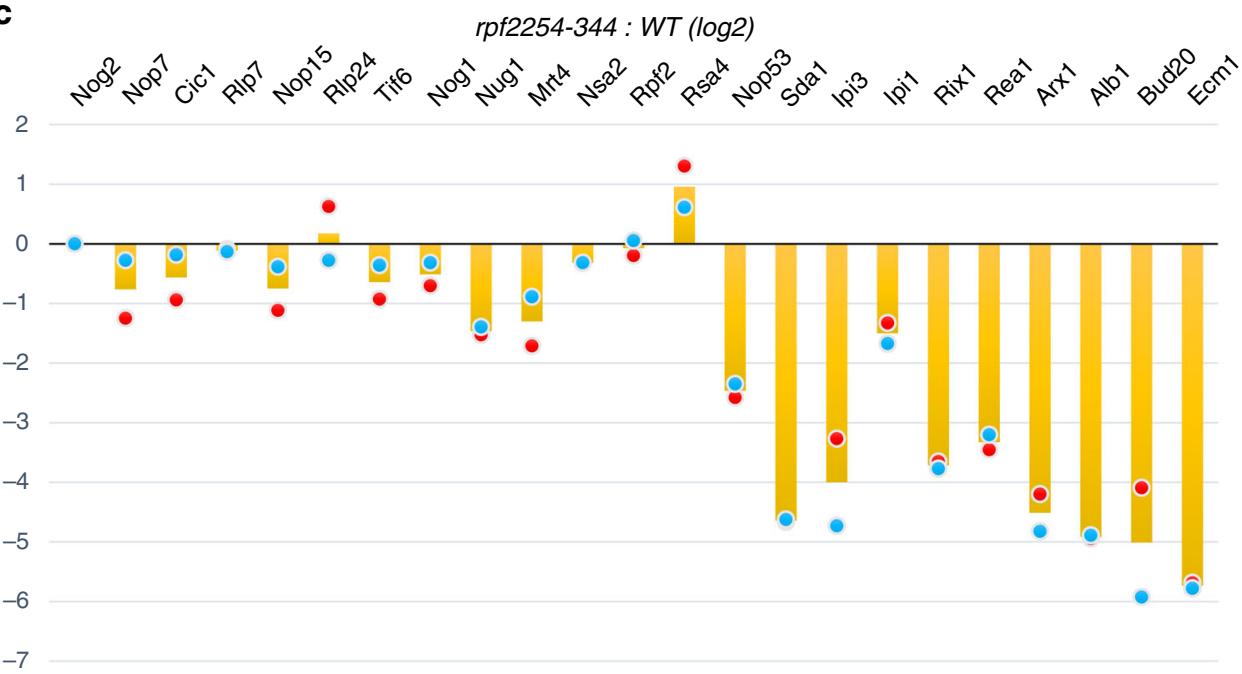

Fig. 2 AFs necessary for $\mathbf{5 S}$ rotation and export are affected in the rpf2 24255-344 mutant. a SDS-PAGE of proteins in assembling 60S subunits from wildtype cells or from the $r p f 2 \Delta 255-344$ mutant shifted to $16^{\circ} \mathrm{C}$ for $5 \mathrm{~h}$. Pre-ribosomal particles were purified using AF Nog2 as a bait and protein constituents were separated by electrophoresis and stained with silver. Molecular weight standards, the Nog2 bait protein Nog2-Cbp (calmodulin-binding peptide left behind after TEV cleavage), and relevant AFs are labeled. b Samples from a were subjected to western blotting using antibodies against specific proteins. All samples were derived from the same experiment and western blottings were processed in parallel. c Samples prepared as described in a were used for iTRAQ (semi-quantitative mass spectrometry) to quantify relative changes in levels of AFs in the genomic rpf2 $255-344$ mutant compared with the wildtype strain. The ratios were normalized to levels of Nog2 (bait). The fold change is shown using bar graphs (orange) in log2 scale as an average of two biologically independent samples $(n=2)$ for the genomic rpf2 $\Delta 255-344$ mutant compared with the wild-type grown at $16^{\circ} \mathrm{C}$. Dot blots represent values for each biological replicate (red and blue). Source data are provided as a Source Data file.

also diminished, consistent with our observation that the mutant particles were not export competent (Fig. 2 and Supplementary Fig. 5a). Levels of Nop53 were also decreased in the $r p f 2 \Delta 255-344$ mutant particles. As Nop53 recruits the exosome machinery to ITS2, processing of this spacer RNA may be affected in some assembling $60 \mathrm{~S}$ particles lacking Nop53 (Fig. 2a, c) ${ }^{29,38}$.

Cryo-EM of $r p f 2 \Delta 255-344$ reveals a block in 5S RNP rotation. To assess changes in the structure of assembling large subunits, we performed cryo-EM analysis of pre-60S particles purified from the rpf2 $\triangle 255-344$ mutant strain. After several rounds of threedimensional (3D) classification, we obtained three major structural classes (C1 to $\mathrm{C} 3,3.9-6.0 \AA$ resolution) (Supplementary Fig. 6). Highly similar classes, $\mathrm{C} 2$ and $\mathrm{C} 3$, were combined for further refinement, providing an improved map (C4, 3.2 $)$. Next, we built and refined an atomic model for the class $\mathrm{C} 4$ particles.

Our cryo-EM results show that in all $r p f 2 \Delta 255-344$ particles, densities for the truncated CTD of Rpf2 were missing, as expected 
a

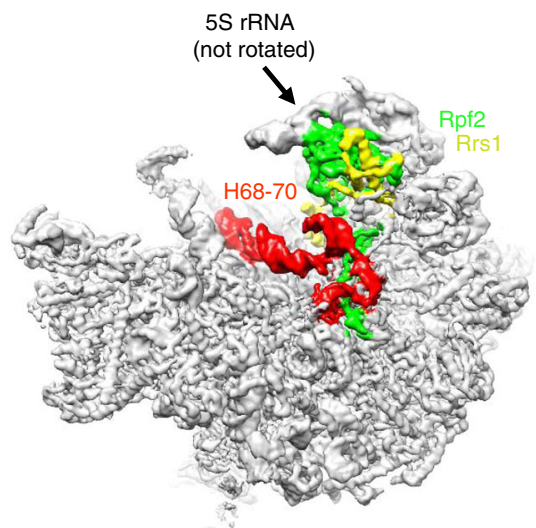

Density map of WT particle

b

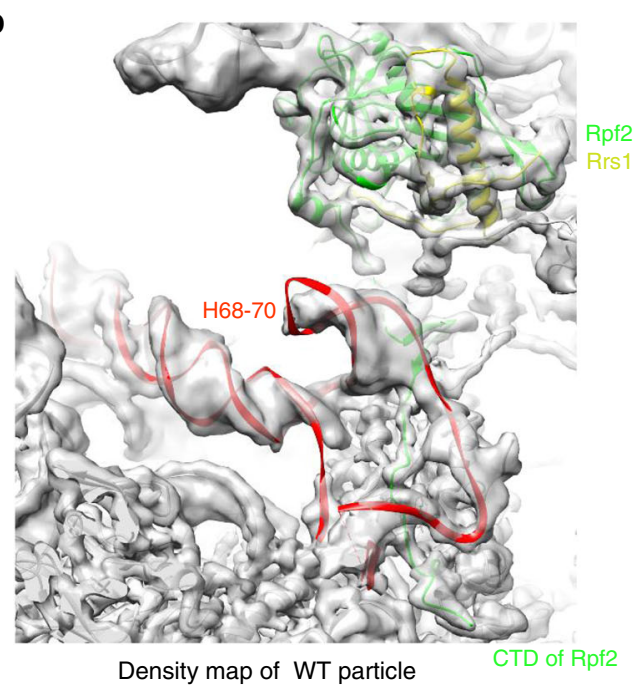

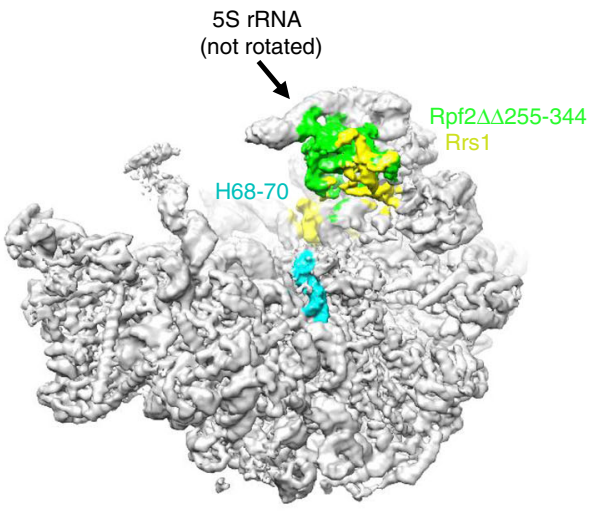

Density map of C4 particle

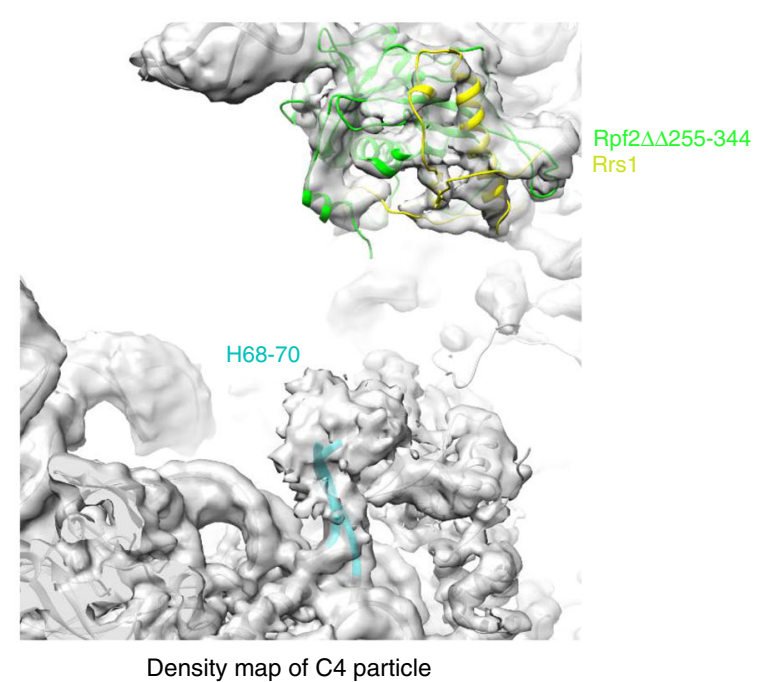

C
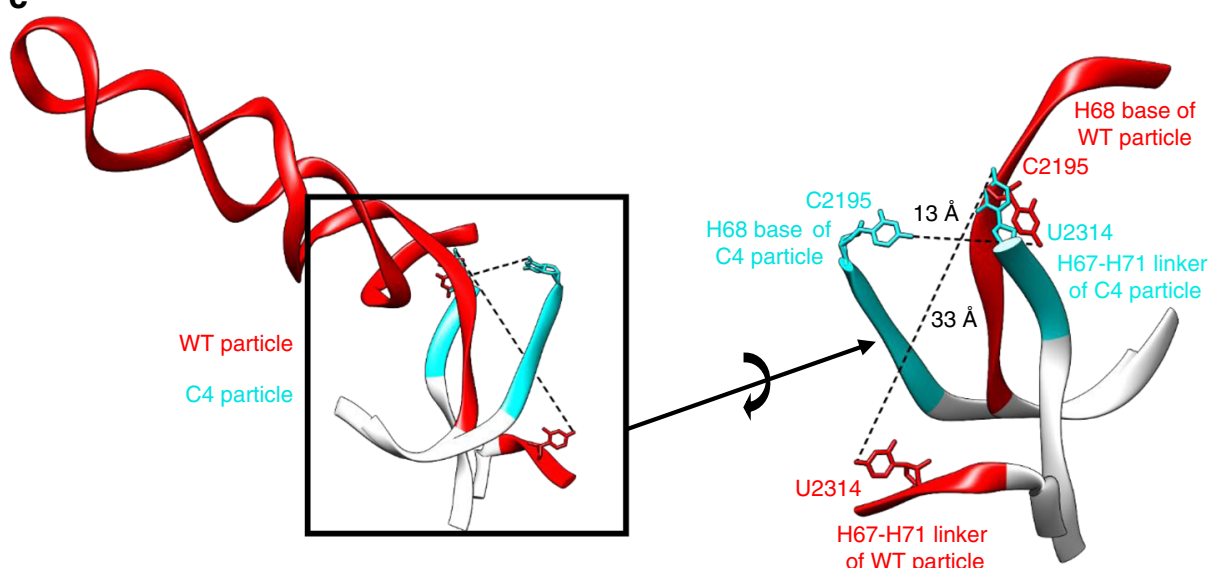

Fig. 3 H68-70 are flexible and invisible in the absence of the CTD of Rpf2. a Subunit interface view of density maps from the wild-type particles and the C4 class of rpf2 255-344 mutant particles. Densities for Rpf2, Rrs1, and H68-70 were colored using Color Zone in Chimera. b Atomic models for wild-type and C4 particles were fitted into density maps of wild-type and C4 particles, respectively. The C4 class of the rpf2 $2255-344$ particles is missing densities for the CTD of Rpf2 and for H68-H71, except for the base of $\mathrm{H} 68$ and the H67-H71 linker. c Structural comparison of atomic models of the wild-type particle and mutant $\mathrm{C} 4$ particle in the stem base region of $\mathrm{H} 68$. One strand of the base of $\mathrm{H} 68$ in the $\mathrm{C} 4$ mutant is deflected up to $13 \AA$, as indicated by the displacement of $\mathrm{C} 2195$, and the $\mathrm{H} 67-\mathrm{H} 71$ linker is deflected up to $33 \AA$.

(Fig. 3). Importantly, in contrast to the wild-type Nog2 sample, in which a small population of particles contains Sda1 (State 2) ${ }^{27}$, no Sda1-containing particles were observed in structural classification of the rpf2 $\Delta 255-344$ mutant pre-ribosomes. Consistent with the absence of Sda1, the 5S RNP was in its pre-rotated state in all classes of mutant particles (Fig. 3a and Supplementary Fig. 6b). Therefore, deletion of the CTD of Rpf2 blocks 5S RNP rotation.

H68-H70 do not fold properly in the $r p f 2 \Delta 255-344$ mutant. A second significant change in the $r p f 2 \Delta 255-344$ mutant particles, 
a

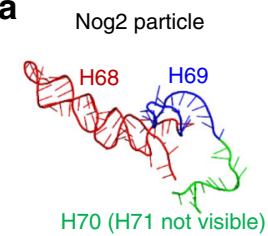
Rix1/Rea1 particle

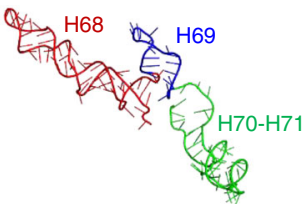

Nmd3 particle

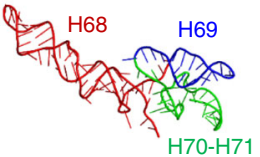

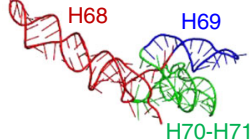

C

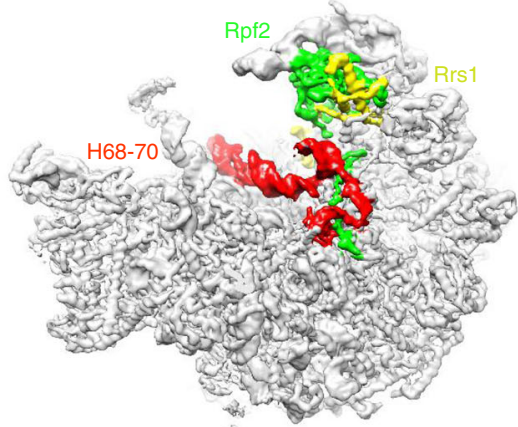

Density map of WT particle

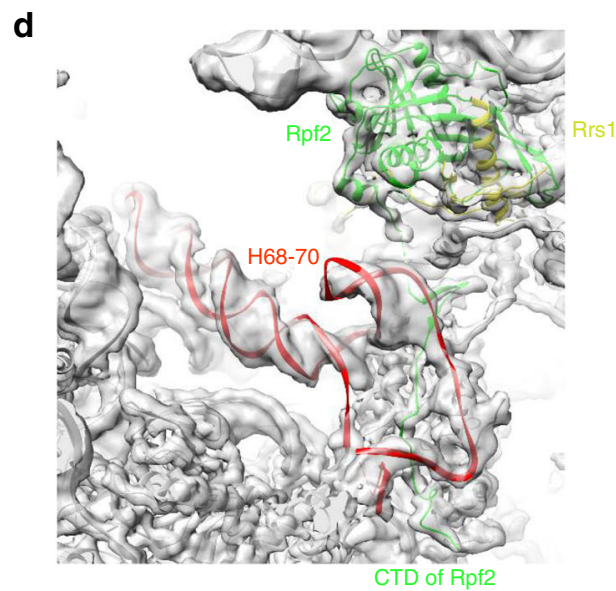

Density map of WT particle b
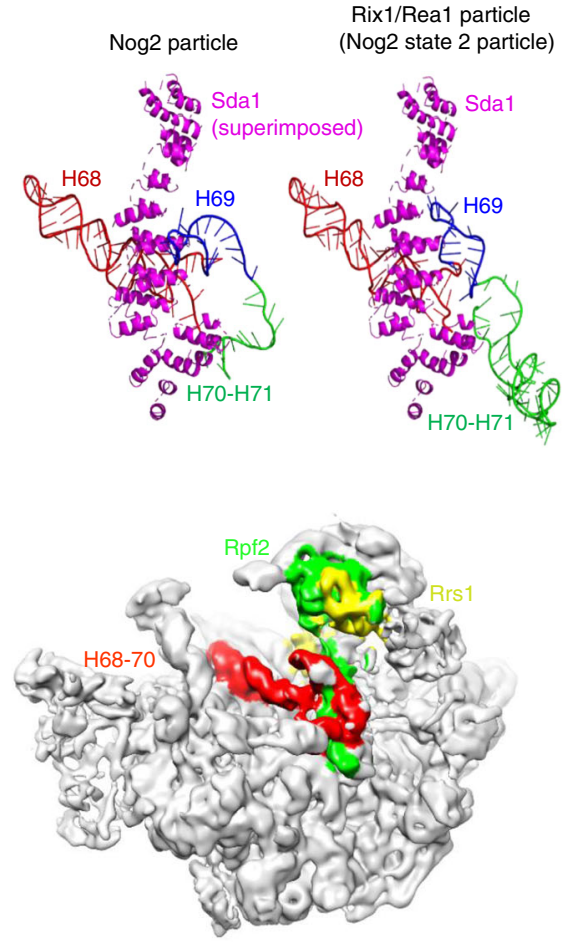

Density map of E2 particle

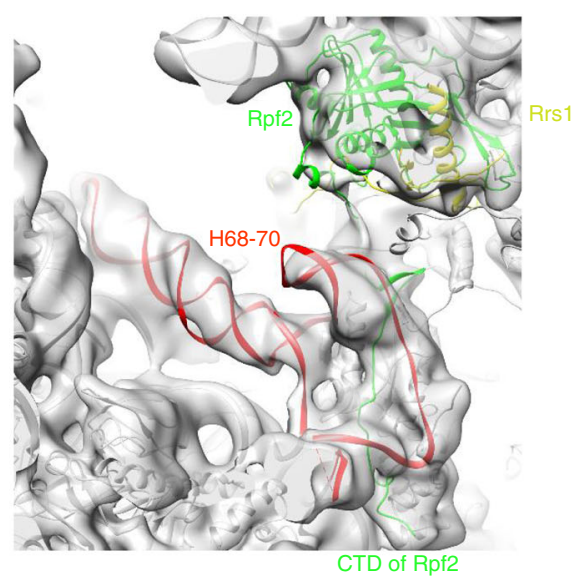

Density map of E2 particle

Fig. 4 Changes in H68-70 are not caused by the absence of Sda1, which binds to H68-69. a Maturation of H68-H71 in wild-type cells during the transition from Nog2 particles to Rix1/Rea1 particles, then Nmd3 particles, and finally to mature 60S subunits (PDB IDs 3JCT [https://www.wwpdb.org/ pdb?id=pdb_00003jct], 5FL8 [https://doi.org/10.2210/pdb5FL8/pdb], 5APN [https://doi.org/10.2210/pdb5APN/pdb], and 4V88 [https://doi.org/ 10.2210/pdb4v88/pdb]). b Left: partial structure of Sda1 from the Rix1/Rea1 (Nog2 state 2) particle superimposed onto the structure of the Nog2 particle (PDB ID 3JCT [https://www.wwpdb.org/pdb?id=pdb_00003jct]). Sda1 is in close contact with $\mathrm{H68}$ and $\mathrm{H69}$ of the Nog2 particle. Right: Sda1 is in close contact with $\mathrm{H} 68$ and H69 in the Rix1/Rea1 particle (PBD ID 5FL8 [https://doi.org/10.2210/pdb5FL8/pdb]), and the conformation of H69 is different from that in the Nog2 particle, presumably due to binding of Sda1. c Subunit interface view of density maps from the wild-type and E2 class of the Sda1-depleted particles. Densities for Rpf2, Rrs1 and H68-70 were colored using Color Zone in Chimera. No densities for H68-70 are missing in the E2 class compared with the wild-type Nog2 densities. d The atomic model for the wild-type particle was fitted into density maps of wild-type and E2 particles, respectively. Densities for Rpf2 and H68-70 are present in particles missing Sda1.

compared with wild-type Nog2 particles, was the absence of densities for rRNA domain IV helices H68-H70 (Fig. 3). In wildtype Nog2 particles, the CTD of Rpf2 is in close contact with these helices (Supplementary Fig. 2d). Helices 68-69 and part of H70 can first be visualized by cryo-EM in Nog2 State 1 particles (H71 is not visible even in that state), but they are still in an immature conformation compared to that in mature $60 \mathrm{~S}$ subunits (Fig. 3a, b left and Fig. 4a). In particular, H69 needs to go through several consecutive steps of structural rearrangements during $60 \mathrm{~S}$ subunit assembly, before it reaches its mature conformation (Fig. 4a and also see "Discussion") 7,9,26,27,39.

Densities for the entire H68-H69 and the visible portion of $\mathrm{H} 70$ were missing in the rpf2 $\Delta 255-344$ mutant (Fig. $3 b$ ). Some densities for the stem base of H68 were still visible (Fig. 3b), but 
significantly deflected compared to wild-type particles (Fig. 3c). These results indicate that H68-70 are not properly folded in mutant particles and explain their invisibility by cryo-EM.

Folding of H68-H70 is not affected by the absence of Sda1. Importantly, one of the AFs that is significantly affected in the rpf2 24244-344 particles, Sda1, binds to rRNA helices H68-H69 in wild-type Nog2 state 2 particles (Fig. $4 \mathrm{~b})^{27}$. Mutation or depletion of Sda1 blocks 60S subunit assembly at the same step as truncation of the CTD of Rpf2 ${ }^{40}$ (Supplementary Fig. 7a-d). As Sda1 does not assemble into pre-ribosomal subunits in the rpf2 $\Delta 255-344$ mutant, the flexible conformation of $\mathrm{H} 68-\mathrm{H} 70$ that we observed in this mutant may be the reason for the absence of Sda1, or else the consequence of its absence. To distinguish between these possibilities, we examined the structures of assembling pre-60S subunits upon directly depleting Sda1. We obtained two refined classes of particles, E1 and E2 (Supplementary Fig. 8). Even though these classes were in many general aspects similar to particles lacking the CTD of Rpf2 (compare Fig. 3a with Fig. 4c), the primary difference was that densities for $\mathrm{H} 68$ - $\mathrm{H} 70$ were not missing when Sda1 was depleted and the conformations of $\mathrm{H} 68$, $\mathrm{H} 69$, and the visible part of $\mathrm{H} 70$ in the $\mathrm{E} 2$ class matched those in wild-type Nog2 particles (Fig. 4d). Thus, improper folding of H68$\mathrm{H} 70$ is likely the reason for the absence of Sdal in the rpf2 2255 344 mutant particles, not the consequence.

The absence of the Rpf2 CTD affects H75 and AFs Nog2 and Rsa4. To assess changes in conformations of pre-rRNAs and proteins, we aligned atomic models of wild-type Nog2 particles and the C4 class of the rpf2 $2255-344$ mutant Nog2 particles. The most significant change in the rRNA was the altered configuration of $\mathrm{H} 75$ in the mutant particles (Fig. 5a). This helix was deflected up to $9 \AA$ in the mutant particles compared with its wild-type position (Fig. 5b). This displacement of $\mathrm{H} 75$ results in a steric clash with superimposed H68 (Fig. 5c). Considering that the rRNA helices underneath $\mathrm{H} 75$ remain in a similar conformation as in wild-type particles, it is likely that the conformational change in $\mathrm{H} 75$ in the $\mathrm{C} 4$ structure is a consequence, rather than a cause, of the failed docking of $\mathrm{H} 68$.

Deflected H75 also clashes with the N-terminal extension of Nog2, with which it is in close contact (Fig. 6a). This might account for the observation that densities for the first 17 amino acids of Nog2 were missing in the C4 map (Fig. 6b). In addition, other portions of Nog2, the GTPase domain and the CTD, were in altered positions in the rpf2 $\Delta 255-344$ mutant (Fig. 6b). As the CTD of Rpf2 binds between Nog2 and H69-70, structural changes in the GTPase domain and CTD of Nog2 might be consequences of the absence of the C-terminal extension of Rpf2.

All classes of particles obtained from the rpf2 $2255-344$ mutant were also lacking densities for the N-terminal domain (NTD) of Rsa4, indicating its flexibility (Fig. 7a, b). During early steps of $5 \mathrm{~S}$ RNP rotation, Rsa4 must reposition itself; the signal for repositioning may be coming through interaction of Rsa4 with Nog2. The NTD of Rsa4 and its globular domain are in close contact with Nog2 (Fig. 7c) ${ }^{27}$. In the rpf2 $2255-344$ mutant, the conformation of Nog2 was slightly altered at both points of contact between Nog2 and Rsa4 (Fig. 7c), and might affect conformation of those regions of Rsa4.

The absence of the CTD of Rpf2 may affect NPET maturation. The CTD of the AF Nog1 (amino acids 595-647) occupies the NPET in wild-type Nog2 particles (Fig. 8a), but is absent from the NPET in the preceding (State E) wild-type particles, suggesting that this Nog1 extension is normally inserted into the NPET once Nog2 enters assembling $60 \mathrm{~S}$ subunits ${ }^{9,27}$. The $\mathrm{C} 2$ and $\mathrm{C} 3$ classes of the $r p f 2 \Delta 255-344$ mutant particles are highly similar, except for differences in densities for Nog1 and a few other AFs. Densities for the CTD of Nog1 were missing in the C2 class of rpf2 2255 344 mutant particles (representing $30 \%$ of particles), whereas the CTD of Nog1 was visible in the C3 class (representing $51 \%$ of particles) (Fig. 8). As densities for the CTD of Nog1 were also lacking in class C1 (19\% of particles), these results show that in $49 \%$ of all particles, the CTD of Nog1 could not be stably inserted into the NPET and was in a flexible state. Thus, the rpf2 $2255-344$ mutant is likely to affect maturation of the NPET through the CTD of Nog1. In addition, densities for the CTD of Rlp24 were also very weak in the C2 class (Fig. 8b). Extensions of Nog1 and Rlp24 are in close proximity, and therefore flexibility of the CTD of Nog1 may affect the CTD of Rlp24.

Nuclear export is blocked in the $r p f 2 \Delta 255-344$ particles. Consistent with the lack of densities for the CTD of Nog1, export factor Arx1, which interacts with the CTD of Nog1 at the rim of the tunnel (amino acids 561-584 of Nog1), was missing from the C2 class of $r p f 2 \Delta 255-344$ mutant particles. Another export factor, Bud20, also exhibited a sub-stoichiometric occupancy. This can partially account for the nuclear export block seen in the rpf2 $\Delta 255-344$ mutant strain (Fig. 8b and Supplementary Fig. 5a).

In addition, densities for Cgr1 and Nug1 were partially missing in the C2 class of $r p f 2 \Delta 254-344$ particles (Fig. 8). Interestingly, densities for Cgrl are not always visualized even in wild-type particles, suggesting that Cgrl is loosely bound to the particles and that it may fall off during purification. Lack of the CTD of Rpf2 may further destabilize particles and weaken Cgr1 binding. Cgr1 is implicated in the stabilization of the rotated state of the $5 \mathrm{~S}$ $\mathrm{RNP}^{41}$. Therefore, the absence of this protein would not add to defects in the rpf2 $\Delta 254-344$ mutant strain, as biogenesis in the absence of the CTD of Rpf2 is blocked at the step before 5S RNP rotation. Nug1 is a cation-dependent GTPase thought to be involved in formation of the PTC ${ }^{42}$; the reason for partial absence of its densities is not clear at this time.

In summary, all of these structural data demonstrating the presence or absence of AFs in pre-60S ribosomes are in an agreement with our SDS-PAGE, western blotting, and iTRAQ data.

The $\mathrm{C} 1$ class may represent turnover intermediates. The $\mathrm{C} 1$ class of particles had an unusual configuration, with highly flexible H68-70, largely displaced H75-79, and the absence of the ITS2 spacer RNA and associated AFs (Fig. 9a, b). In addition, the L1 stalk was in a closed position (Fig. 9b, c), sharply different from those observed in wild-type particles (refs. $26,27,43,44$ ); it was deflected toward H81 instead of toward the 5S RNP. This unusual conformation of the L1 stalk resulted from the significant displacement of helices H75-79, which are located at the base of the L1 stalk (Fig. 9b). This $\mathrm{C} 1$ class may represent a turnover intermediate, and is not specific to the rpf2 $2255-344$ mutant strain. This class of particles was also observed upon depletion of Sda1 (with the exception of H69, which was not affected) (class E1, Fig. 9). In the rpf2 $\Delta 255-344$ mutant, the $\mathrm{C} 1$ class accounts for around $19 \%$ of all particles, whereas in the Sda1-depleted strain, $67 \%$ of the particles belong to this class.

\section{Discussion}

Previously, we had shown that AF Rpf2 is necessary for initial docking of the 5 S RNP into maturing 60S subunits ${ }^{20}$. Here we show that it is very unlikely that the CTD of Rpf2 is necessary for docking and early stages of subunit assembly. Instead, it appears that this extension is necessary during middle stages of assembly, more specifically for late stages of anchoring of the Rpf2 subcomplex to pre-60S subunits. We believe that the 

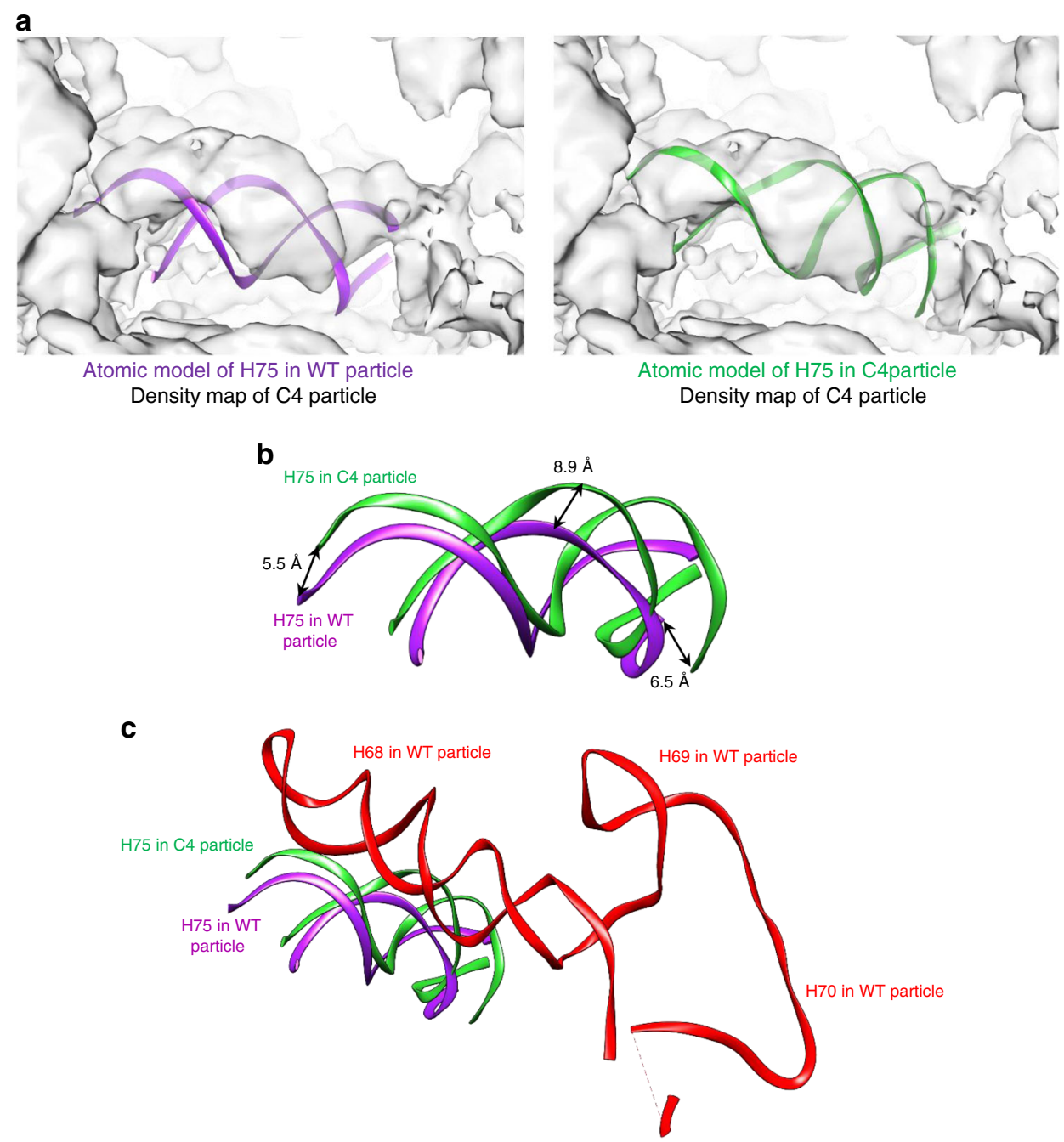

Fig. 5 H75 is deflected in the C4 class of rpf2 24255-344 particles and clashes with H68. a The atomic model for H75 of wild-type particles (left) or the C4 class of rpf2 $255-344$ mutant particles (right), was fitted into the density map of the C4 class of rpf2 $2255-344$ particles. b Atomic models for H75 in wild-type and $\mathrm{C} 4$ particles were aligned. $\mathrm{H} 75$ is deflected up to $8.9 \AA$ in the $\mathrm{C} 4$ class compared with wild-type particles. $\mathbf{c} \mathrm{H} 75$ in the $\mathrm{C} 4$ class of the rpf2 255 -344 particles clashes with the position of $\mathrm{H} 68$ in wild-type particles. $\mathrm{H} 68-\mathrm{H} 70$ are not visualized in the $\mathrm{C} 4$ class due to their flexibility.

primary defect caused by the absence of the long C-terminal extension of Rpf2 is misfolding of rRNA helices H68-H70. This misfolding may be initiated by the loss of interactions between the CTD of Rpf2 and H68-70. Alternatively, it may be caused indirectly, through the adjacent GTPase Nog2, which may be affected by the truncation of the C-terminal extension of Rpf2, or by some other, still undiscovered path. Regardless, any perturbance in the neighborhood of helices H68-70 may prevent conformational maturation of these helices, and most likely cause a block in 60S subunit assembly.

Sda1, necessary for 5S RNP rotation, binds to H68 and H69; therefore, the improper conformation of these helices is likely preventing binding of Sda1. The inability of Sda1 to bind affects association of the Rix1 subcomplex and Rea1, and thus rotation of the 5S RNP (Fig. 10). As a result, rotation of the 5S RNP cannot occur.

In wild-type cells, once the Rpf2 subcomplex is stably anchored (together with rRNA domain V) to the main body of assembling 60 S subunits, $\mathrm{H} 68-\mathrm{H} 70$ can be visualized for the first time by cryoEM in State 1 Nog2 particles, but still in an immature conformation compared to that in mature $60 \mathrm{~S}$ subunits $7,9,26,27,39$. In particular, H69 needs to go through several structural rearrangements during $60 \mathrm{~S}$ subunit assembly before it reaches its mature position
(Fig. 4a). Presumably, these stepwise conformational changes of H69 are mediated at least in part by sequential binding of proximal AFs, including Rpf2, Nog2, Sda1, Nmd3, and Lsg1, and by EngA during bacterial $50 \mathrm{~S}$ subunit assembly $11,19,27,33,44,45$.

H69 is universally conserved. In bacteria, its deletion has a dominant lethal phenotype ${ }^{35,36}$. H69 is an integral part of the PTC. The closing loop of H69 contacts the mRNA decoding center of the small subunit, while its stem forms a portion of the $\mathrm{P}$ site in the large subunit. H69 is a critical part of the B2a intersubunit bridge that communicates with h44 of the small ribosomal subunit ${ }^{34,35}$. In mature subunits, $\mathrm{H} 69$ is a potent regulator of translation as it alters small subunit rotation dynamics ${ }^{36}$. Considering the importance of $\mathrm{H} 69$, it is clear why mechanisms have evolved to keep this helix in an immature conformation until the $60 \mathrm{~S}$ subunit is fully formed. Thus, the controlled maturation of $\mathrm{H} 69$ is essential for both proper formation and function of $60 \mathrm{~S}$ subunits.

$\mathrm{H71}$, similar to H69, goes through significant rearrangements during 60 S subunit assembly (Fig. 4a) ${ }^{19,27}$. However, H71 is not yet visible in Nog2 particles, which prevents us from ascertaining whether this helix is also affected in the $r p f 2 \Delta 255-344$ mutant strain.

$\mathrm{H} 75$ is significantly deflected from its native position in rpf2 $\Delta 255$-344 mutant particles (Fig. 5a, b). The flexibility of H68 
a

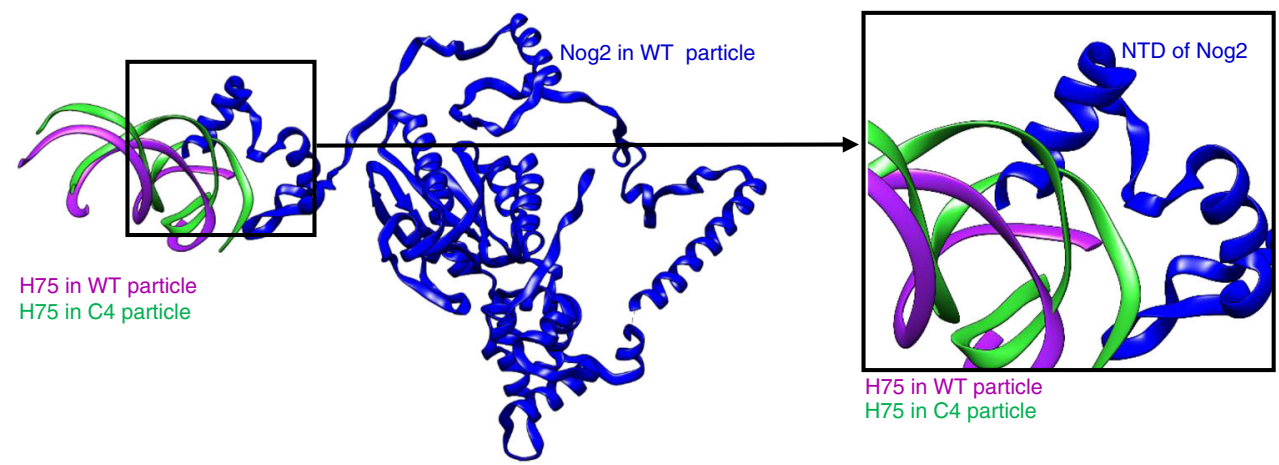

b

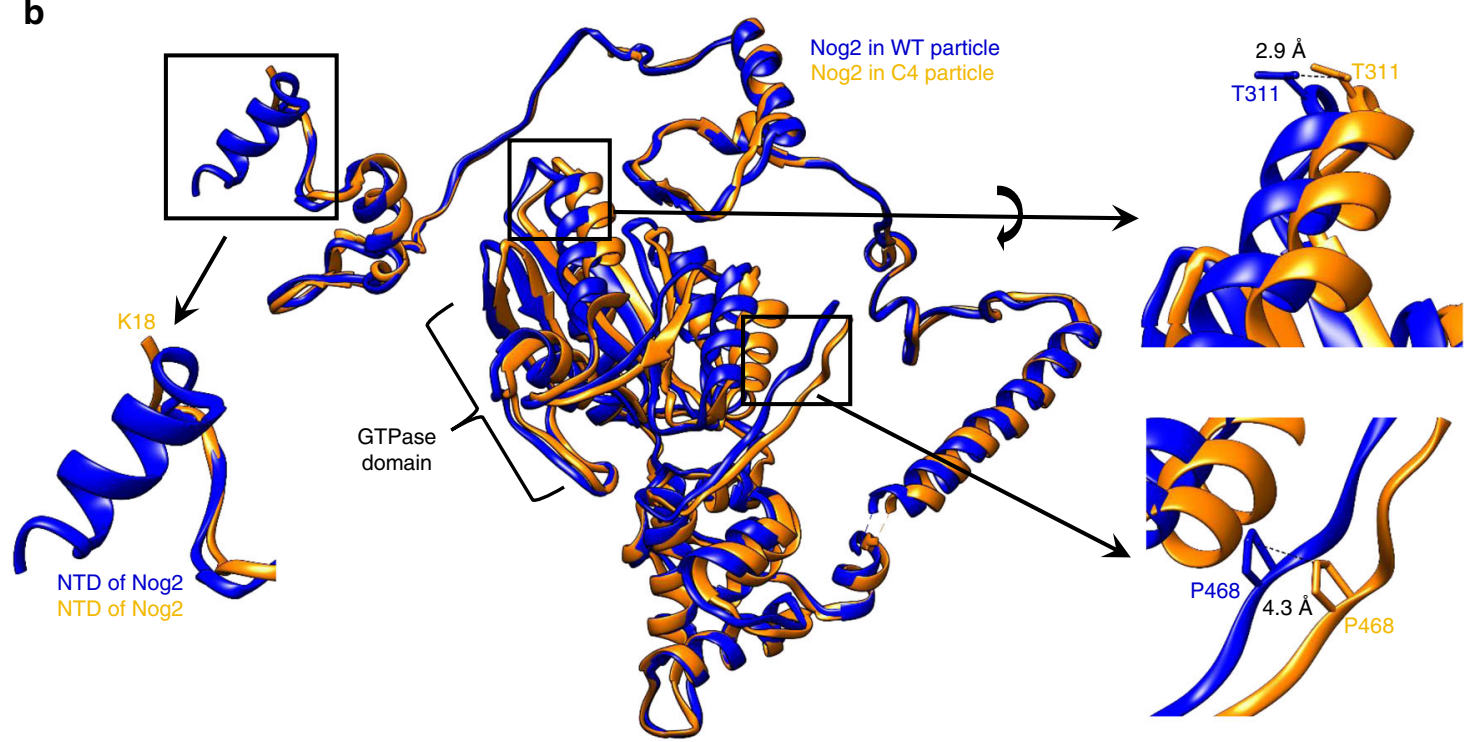

Fig. 6 The conformation of Nog2 is altered in the C4 class of rpf2 255-344 particles. a The NTD of Nog2 is docked in the groove of H75 near H68 in wild-type particles and may affect the conformations of these helices, or otherwise be affected by them. $\mathbf{b}$ Atomic models for Nog2 in wild-type and C4 particles were aligned. The NTD of Nog2 is flexible and invisible in the C4 class of the rpf2 $2255-344$ mutant particles. Conformational changes are visible in the GTPase domain of Nog2, where portions of the structure are deflected up to $4 \AA$.

is likely destabilizing $\mathrm{H} 75$ and allowing its deflection compared with wild-type particles (Fig. 5c). As the N-terminal extension of Nog2 is docked in the groove of H75, its flexibility may affect the conformation of $\mathrm{H75}$, or vice versa (Fig. 6a).

Other conformational changes in Nog2 affect its GTPase and CTD domains. Points of contact among the CTD of Rpf2, H68H70, and Nog2 are close to the Nog2 GTPase domain (Supplementary Fig. 1b, c). This may explain the phenotype of the rpf2-2 mutant (Fig. 1a and Supplementary Fig. 7e-g), mutations in which target interactions of the Rpf2 extension with the Nog2 GTPase domain. These mutant amino acids can also affect interactions between the Rpf2 CTD and H68-70, since they are in close proximity to junctions of these helices. The altered configuration of Nog2 might affect the conformation of H68-70, or vice versa, the conformation of Nog2 may be affected by these RNA helices. The absence of any of these interactions may affect Nog2 in a way that prevents activation of its GTPase activity and exit from assembling $60 \mathrm{~S}$ subunits.

In the absence of the CTD of Rpf2, the CTD of Nog1 does not enter the NPET in $49 \%$ of particles. It is currently unclear what the signal is for entry of this extension of Nog1 into the NPET. However, it has been proposed that the NPET and PTC may communicate with each other ${ }^{46}$. Thus, the incorrect conformation of H68-70 may be transmitted to the NPET (possibly through H75) and prevent further steps in NPET maturation (such as insertion of the Nog1 CTD) through steric changes in rRNA conformation. Alternatively, the block caused by the absence of the CTD of Rpf2 may block assembly upstream of entry of the Nog1 CTD into the NPET.

Processing of the ITS2 spacer RNA seems to proceed normally in at least some portion of these mutant particles, consistent with previous reports that 5S RNP rotation and ITS2 removal can occur independently from each other ${ }^{41,47-49}$. In the majority of particles, however, the block caused by truncation of the Cterminal extension of Rpf2 likely halts subunit assembly before the processing of ITS2 would normally occur. That is probably the reason why most particles still contain densities for ITS2 and its associated proteins.

Anchoring of $25 \mathrm{~S}$ rRNA domain $\mathrm{V}$ and the Rpf2 subcomplex containing the 5S RNP can only occur after (1) Rea1 removes Ytm1 and Erb1, (2) Has1, Drs1, Nop2, Nip7, Ebp2, Brx1, Spb1, and Noc3 dissociate from assembling subunits, and (3) H74-79 anchor to the main body of the subunit (Fig. 10$)^{6,9}$. We propose that the signal that stable anchoring of the Rpf 2 subcomplex has occurred is transmitted through the CTD of Rpf2. The CTD of Rpf2 stabilizes H69 in a structurally defined intermediate conformation, and the subsequent binding of Sda1 rearranges H69 into its next conformation (Fig. 4a, b). Thus, the absence of the CTD of Rpf 2 may prevent $\mathrm{H} 69$ from reaching its stable intermediate conformation, and may prevent $\mathrm{H} 68$ from reaching the near-mature conformation 

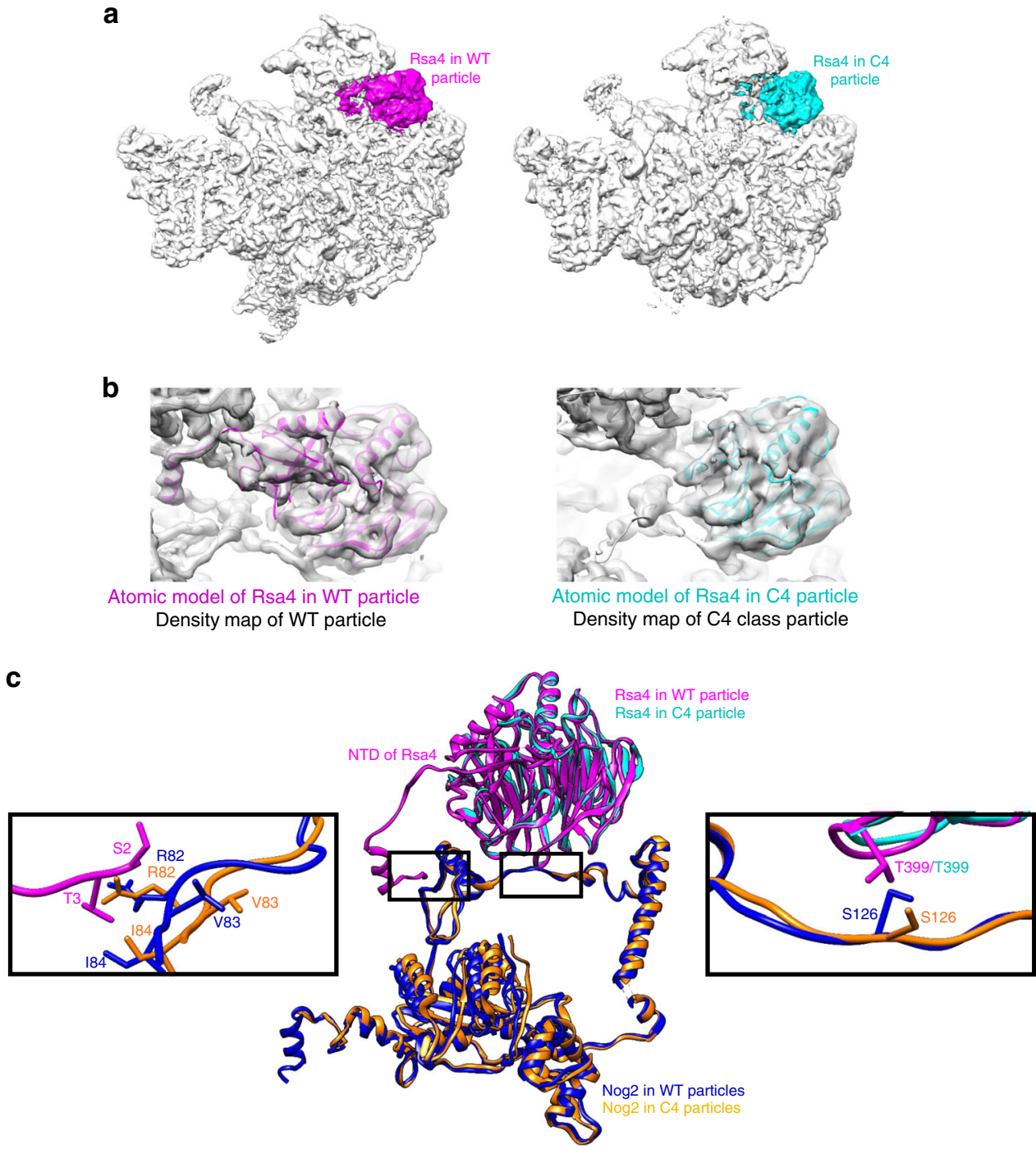

Fig. 7 The NTD of Rsa4 is invisible, and likely flexible in the C4 class of $r$ pf2 $2 \Delta$ 255-344. a The subunit interface view of density maps from the wild-type RPF2 and the C4 class of rpf2 255-344 mutant particles. Densities for Rsa4 were colored using Color Zone in Chimera. b Atomic models for Rsa4 from the wild-type and C4 class of the rpf2 $255-344$ mutant were fitted into density maps of the wild-type and the C4 class mutant. The first 34 amino acids of the NTD of the Rsa4 are flexible and invisible in the absence of the CTD of Rpf2. c Atomic models for Rsa4 and Nog2 from the wild-type and the C4 class are colored and labeled. For clarity, only relevant side chains are shown. Conformational changes in Nog2 may affect Rsa4, because Nog2 and Rsa4 are in close contact at two points (rectangles): at the NTD and the globular domain of Rsa4. Enlarged rectangles show contact points between Rsa4 and Nog2.

needed for Sdal binding. Overexpression of Sda1 failed to suppress the slow growth phenotype of the $r p f 2 \Delta 255-344$ mutant strain at $16^{\circ} \mathrm{C}$ (Supplementary Fig. 7h), indicating that the block caused by the absence of Rpf2 occurs upstream of the entry of Sda1 into assembling $60 \mathrm{~S}$ subunits.

The yields of pre-ribosomes purified from the rpf2 $\Delta 255-344$ mutant strain are slightly lower than those from the wild-type strain. This suggests that a small percentage of particles are turned over in the rpf2 $2255-344$ mutant strain before they could be detected by SDS-PAGE or visualized by cryo-EM. In addition, it is likely that the "bad particles" that we observed by cryo-EM in this mutant are destined for turnover. We speculate that the reason for this turnover is the unfolding of H68-70 in the absence of the CTD of Rpf2, and that this specific rRNA unfolding may trigger the turnover machinery. This explains why these bad particles are not as prevalent in the strain in which Sdal is depleted, as H68-70 are not misfolded in that strain.

In summary, 5S RNP rotation appears to be coupled with maturation of functional centers during middle stages of large ribosomal subunit assembly. The nature of this coupling is not yet clear, but it is likely that H68-70 play a crucial role in it. When anchoring of the Rpf2 subcomplex occurs properly, a signal may propagate through the maturing subunit via the C-terminal extension of Rpf2 to Nog2 and H68-H70. Since conformational maturation of $\mathrm{H68-70}$ is a prerequisite for entering the next major stages of subunit biogenesis, 5S RNP rotation and nuclear export, the failure of $\mathrm{H} 68-\mathrm{H} 70$ to fold properly triggers a series of downstream events that block progression of $60 \mathrm{~S}$ subunit biogenesis, including NPET maturation and PTC assembly. Therefore, we speculate that conformational maturation of $\mathrm{H} 68-70$ plays a critical role in the middle steps of assembly and that proper folding of these helices is necessary not only for function of the mature 60S subunit, but is also vital during its biogenesis.

\section{Methods}

Construction of yeast strains and mutagenesis. Yeast strains and plasmids used in this study are listed in Supplementary Tables 1 and 2. A yeast strain conditional for expression of RPF2 was constructed by replacing the endogenous promoter of 
a

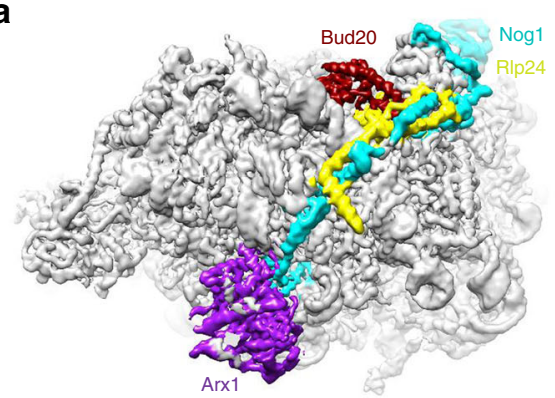

WT particle (bottom view at NPET)

b

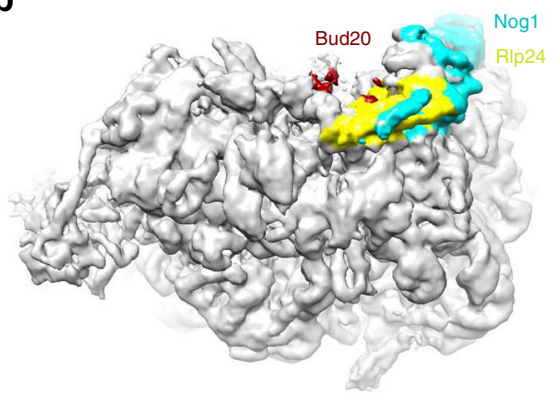

C2 particle (bottom view at NPET)

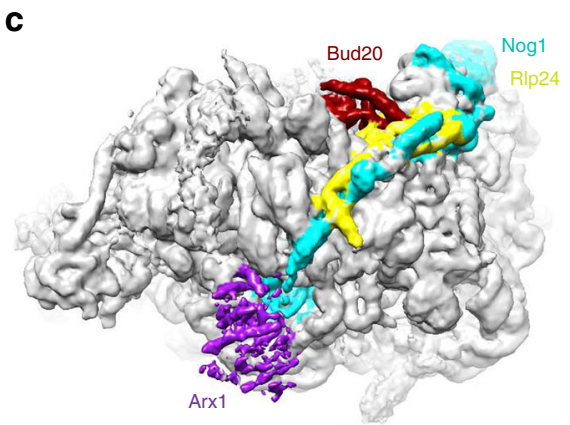

C3 particle (bottom view at NPET)

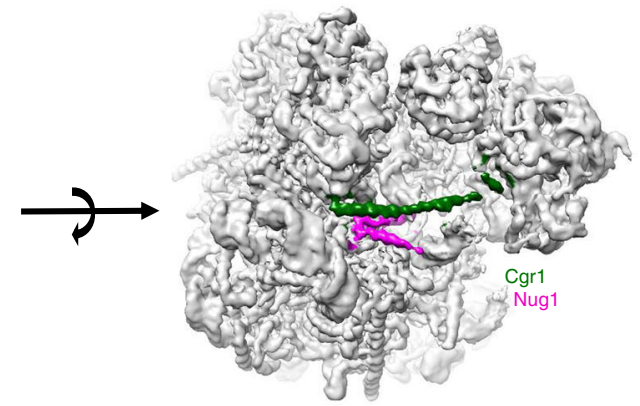

WT particle (side view)

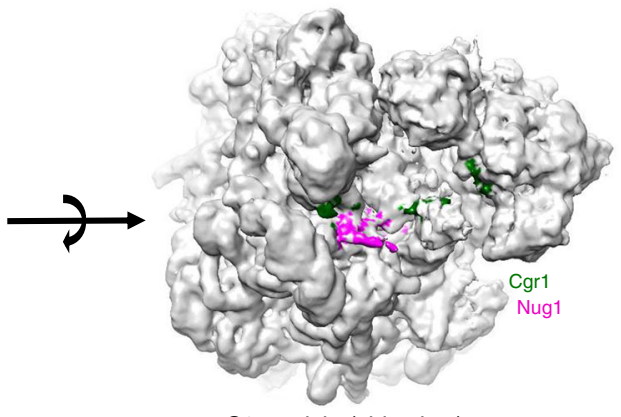

C2 particle (side view)

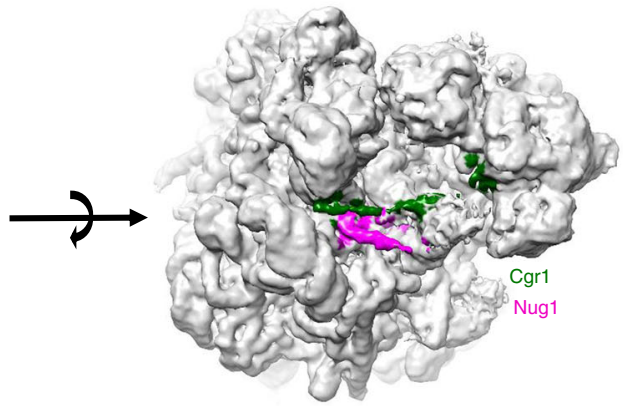

C3 particle (side view)

Fig. 8 NPET maturation and nuclear export are affected in the rpf2 2555-344 mutant. a Subunit interface view (left) and side view (right) of the density map of wild-type particles. Densities for Nog1, Arx1, Bud20, Rlp24, Nug1, and Cgr1 were colored using Color Zone in Chimera. b Same as in a for the C2 class of rpf2 255-344 mutant particles. Densities for the CTD of Nog1, all of Arx1, the CTD of Rlp24, and portions of Bud20, Nug1, and Cgr1 are absent in the C2 class. c Same as in a for the C3 class of rpf2 $255-344$ mutant particles. Densities for Nog1, Arx1, Rlp24, Bud20, Nug1, and Cgr1 are present in the C3 class of $r p f 2 \Delta 255-344$ mutant particles.

$R P F 2$ with the galactose-inducible, glucose-repressible GAL1 promoter including an in-frame insertion of the triple hemagglutinin (3HA) epitope. The template used for PCR was pFA6a-TRP1-pGAL1-3HA ${ }^{50}$. Sequences of oligonucleotides used for the PCR are available upon request. The correct size for the PCR product was confirmed by agarose gel electrophoresis. PCR products were purified and trans formed into a wild-type strain, and the transformants were selected on C-trp + gal solid media. Replacement of the promoter was confirmed by colony PCR

(sequences of oligonucleotides used are available upon request), and expression of the 3HA epitope in frame with the RPF2 was confirmed by western blotting using anti-HA antisera (Thermo Fisher Scientific). The GAL-SDA1 strain conditional for expression of Sdal was a gift from Dr. Douglas R. Kellogg. Strains expressing TAPtagged Nop7 or Nog2 were generated by replacing the stop codon of either NOP7 or NOG2 with the TAP-tag. The TAP-tag plasmid ${ }^{51}$ was used to amplify the TAPtag and sequences immediately upstream or downstream of stop codons (sequences of oligonucleotides are available upon request). The correct sizes of the PCR products were confirmed by agarose gel electrophoresis, PCR products were purified and transformed into wild-type or GAL-RPF2 strains. Transformants were selected on C-ura or C-ura+gal solid media. The presence of the C-terminal tag was confirmed by colony PCR (sequences of oligonucleotides used are available upon request), and the expression of the tagged protein by western blotting using anti-TAP antisera (Promega).

A strain expressing the Rrs1-3HA protein was generated by replacing the stop codon of RRS1 by the 3HA sequence in frame. The plasmid pFA6a-3HA-HIS3MX6 ${ }^{50}$ was used to amplify the 3HA tag and sequences immediately upstream or downstream of the stop codon of RRS1 (sequences of oligonucleotides are available upon request). The correct size of the PCR product was confirmed by agarose gel electrophoresis, and the PCR products were purified and transformed into the GALRRF2 NOP7-TAP strain. Transformants were selected on C-his+gal solid media. The presence of the C-terminal tag was confirmed by colony PCR (sequences of oligonucleotides used are available upon request), and the expression of the tagged protein by western blotting using anti-HA antisera (Thermo Fisher Scientific).

A plasmid expressing Rpf2 was generated by cloning the open reading frame plus 700 nucleotides upstream and 150 nucleotides downstream into the pRS315 plasmid at the multiple cloning site. Correct in-frame insertion was confirmed by sequencing (Genewiz).

Mutations and truncations in RPF2 (rpf2 $2555-344, r p f 2 \Delta 310-344, r p f 2-1$, and $r p f 2-2)$ were introduced into the pRS315-RPF2 plasmid, using the QuikChange II Site-directed mutagenesis kit (Agilent), as per the manufacturer's protocol.

Mutations were confirmed by sequencing (Genewiz). Sequences of oligonucleotides are available upon request.

Plasmids expressing MYC-tagged versions of RPF2 and rpf2 $2255-344$ were generated by Epoch Life Science, Inc. Sequences for two Myc tags were inserted at the $5^{\prime}$-end of either gene in the pRS315 vector and correct in-frame insertion was confirmed by sequencing.

A diploid strain heterozygous for the $r p f 2 \Delta 255-344$ mutant allele expressing a truncation of the CTD of Rpf2 was constructed by truncating one of the alleles of 
a

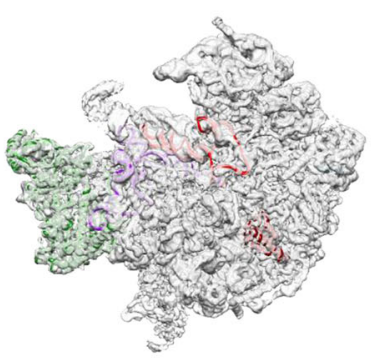

WT particle

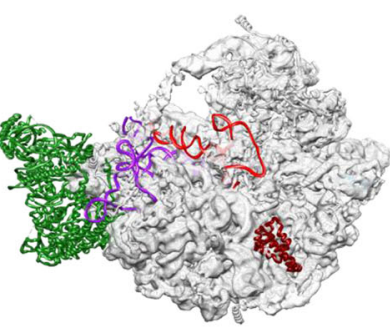

C1 particle

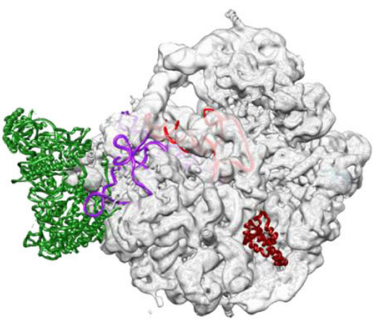

E1 particle b

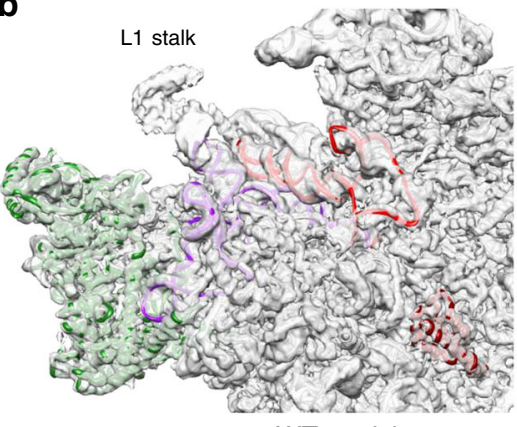

WT particle

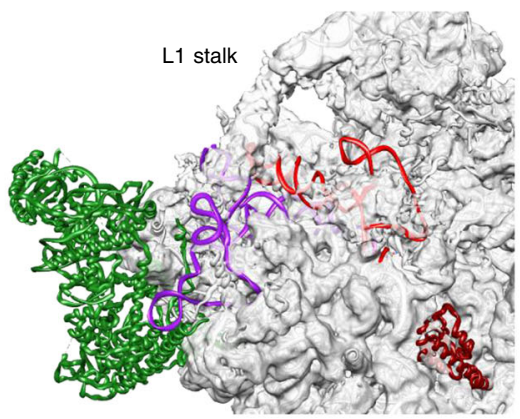

C1 particle

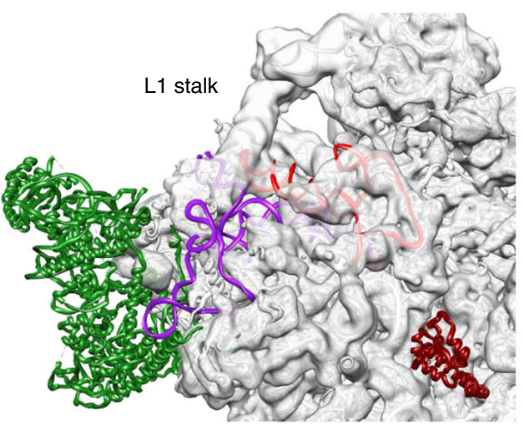

E1 particle
C

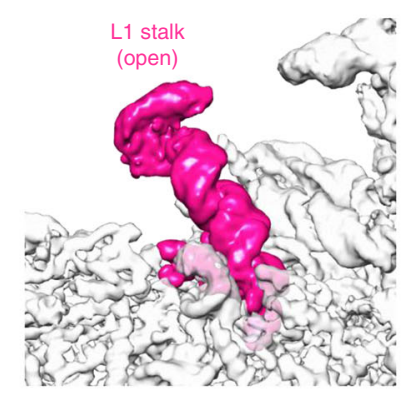

WT particle

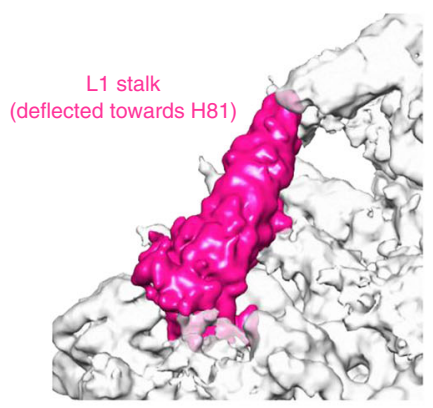

C1 particle

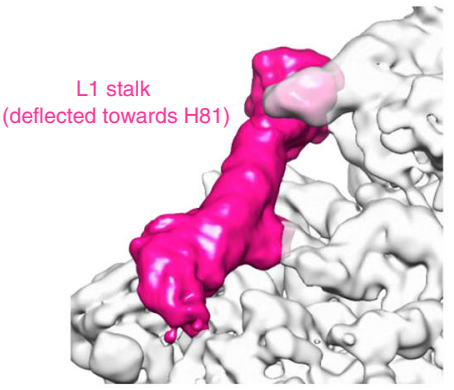

E1 particle

Missing densities for ITS2,

Rip7, Cic1, Nop7, and Nop15

Missing densities for $\mathrm{H} 68-\mathrm{H} 70$

Missing densities for $\mathrm{H} 75-79$

Missing densities for Bud20

Fig. 9 The C1 and E1 classes are unusual and presumably destined for turnover. a The subunit interface view of density maps from wild-type particles, the C1 class of rpf2 $255-344$ mutant particles, and the E1 class of particles from Sda1-depleted yeast. The atomic model for wild-type particles was fitted into density maps of the wild-type and E1 class of mutant particles. The atomic model for the C4 class of mutant particles was fitted into the $\mathrm{C} 1$ class of mutant particles. b Enlarged view of the density maps from a. Densities for the ITS2 spacer rRNA and associated AFs RIp7, Cic1, Nop7, Nop15, and Nop53 (all green), rRNA helices H75-79 (purple), and Bud2O (brown) are missing in the C1 and E1 classes. H68-70 (red) are missing in the C1 class, but are not significantly affected in the E1 class of mutant particles. (c) Enlarged portion of the density maps from (a). The L1 stalk (colored in pink) is in an unusual position in the $\mathrm{C} 1$ and $\mathrm{E} 1$ classes of particles, deflected toward $\mathrm{H} 81$.

the RPF2 by homologous recombination of the PCR product lacking codons for amino acids 255-344 and containing sequences immediately upstream and downstream from them. The plasmid used as a template was pFA6a-kanMX $6^{50}$. Sequences of oligonucleotides are available upon request. The correct size of the PCR product was confirmed by agarose gel electrophoresis, and the PCR products were purified and transformed into the wild-type strain. Transformants were selected on YEPD + G418 solid media. Diploid candidates were sporulated at $30^{\circ} \mathrm{C}$ and tetrads were dissected. Two out of four tetrads were very slow growing. The absence of the C-terminal extension of Rpf 2 in slow growing tetrads was confirmed by colony PCR and sequencing of the PCR product that amplified the genomic region of the truncation. Sequences of oligonucleotides used are available upon request. As the absence of the Rpf2 CTD causes a growth defect even at $30^{\circ} \mathrm{C}$, we used the wild-type RPF2 strain shifted to $16^{\circ} \mathrm{C}$ as a control in our experiments.
Growth of yeast strains. Yeast strains were grown at $30{ }^{\circ} \mathrm{C}$ in either YEPGal $(2 \%$ galactose, $2 \%$ peptone, and $1 \%$ yeast extract) or YEPGlu (2\% dextrose, $2 \%$ peptone, and $1 \%$ yeast extract). The conditional strains containing plasmids were grown in selective media (C-leu + gal) containing galactose and were shifted to selective media $(\mathrm{C}-\mathrm{leu}+\mathrm{glu})$ containing glucose for $17 \mathrm{~h}$. The genomic mutant rpf2 2255 344 strain and its corresponding wild-type strain were grown in YEPGlu media at $30^{\circ} \mathrm{C}$ and shifted to $16^{\circ} \mathrm{C}$ for $5 \mathrm{~h}$.

Sucrose gradient assays of ribosomes and polyribosomes. Cultures $(100 \mathrm{ml})$ of $R P F 2-T A P$ and RRS1-TAP strains were grown in YEPGlu liquid media at $30^{\circ} \mathrm{C}$ to an $\mathrm{OD}_{600}$ of 0.7. Cycloheximide $(5 \mathrm{mg})$ was added to cultures $30 \mathrm{~min}$ before collecting cells. Extracts were made using lysis buffer (10 mM Tris- $\mathrm{HCl}$ pH 7.5, $0.1 \mathrm{M}$ $\mathrm{NaCl}, 30 \mathrm{mM} \mathrm{MgCl}_{2}, 50 \mu \mathrm{g} / \mathrm{ml}$ cycloheximide, $200 \mu \mathrm{g} / \mathrm{ml}$ heparin, and $0.2 \%$ diethyl 
b

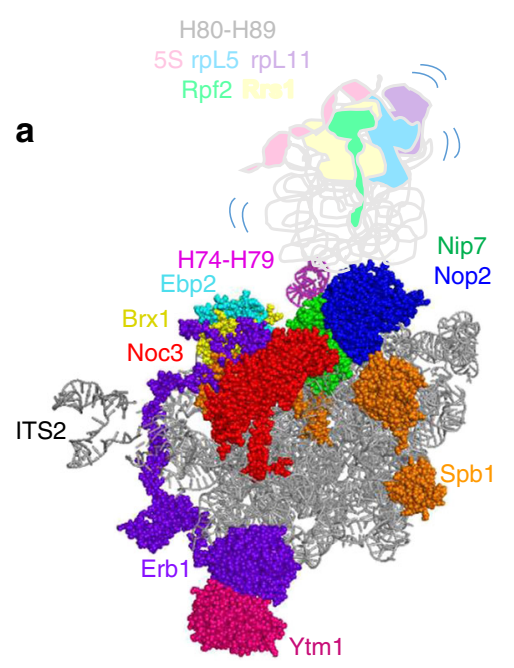

State E particle

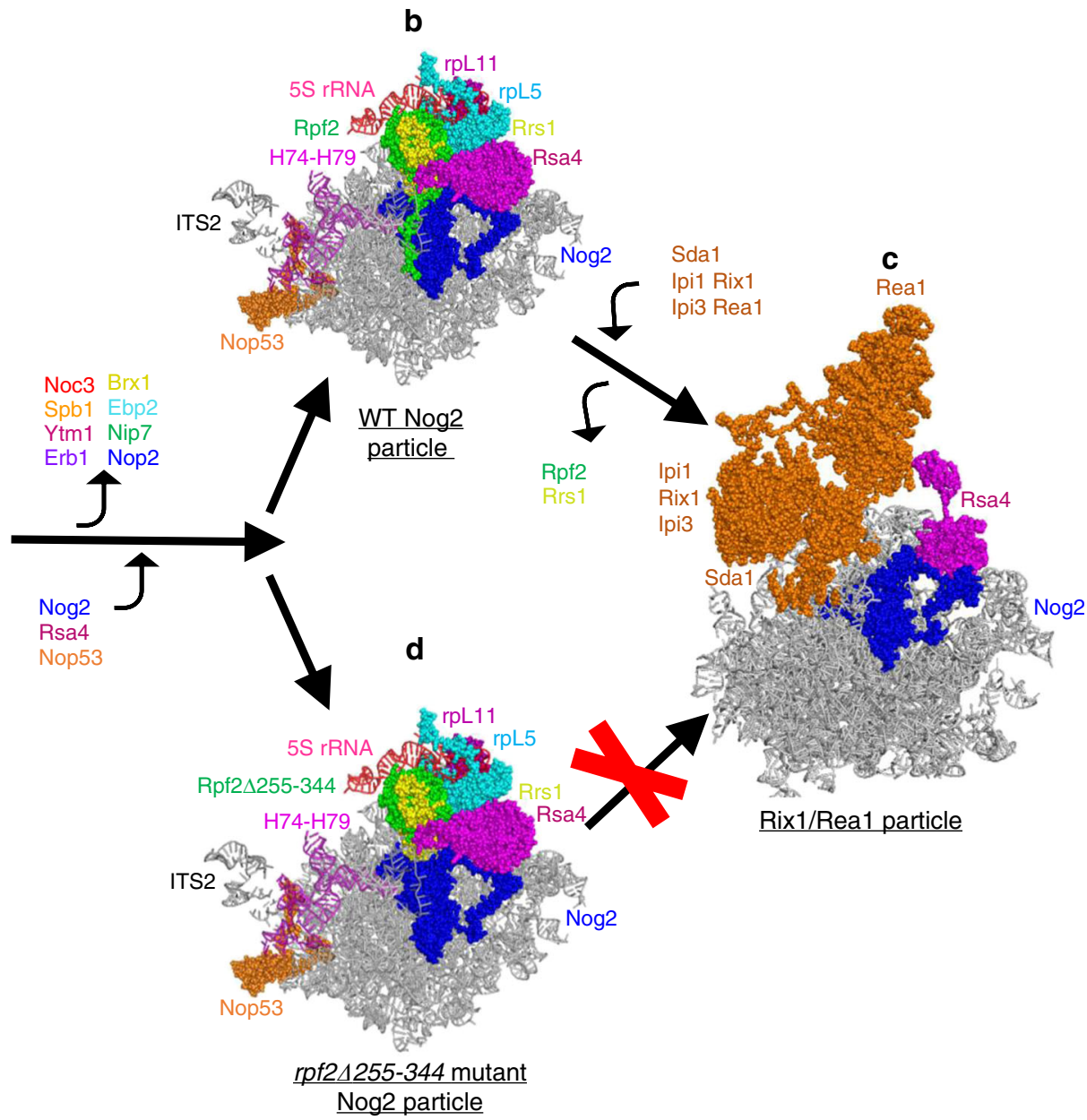

Fig. 10 The CTD of Rpf2 is necessary as Nog2 particles transition to Rix1/Rea1 particles. a The $25 \mathrm{~S}$ rRNA domain $\mathrm{V}$ and the Rpf2 subcomplex are in a flexible state and thus invisible (represented as the group in light colors) prior to rearrangements within the State E particle, including exit of labeled AFs from state E particles. b Stable anchoring of $\mathrm{H} 74-\mathrm{H} 79$, conformational changes in $\mathrm{H} 68-70$, as well as entry of Nog2, Rsa4, and Nop53, upon formation of Nog2 particles. c The Rix1/Rea1 particle is formed upon exit of the Rpf2-Rrs1 dimer, and entry of Sda1, the Rix1 subcomplex, and Rea1, as well as 5S RNP rotation. $\mathbf{d}$ In the absence of the CTD of Rpf2, the transition between the Nog2 and Rix1/Rea1 particles does not occur. For simplicity and clarity, only relevant AFs are shown and colored, and all r-proteins except rpL5 and rpL11 are omitted. Only partial densities for Sda1, Rea1, and the Rix1 subcomplex are available.

pyrocarbonate). Cells were vortexed eight times for $30 \mathrm{~s}$ with glass beads $(0.5 \mathrm{~mm}$ diameter, Biospec Products), kept on ice in between vortexing, and clarified by two consecutive centrifugations. Forty $\mathrm{OD}_{254}$ units of whole-cell extracts were layered on $7-47 \%(\mathrm{w} / \mathrm{v})$ sucrose gradients, and pre-ribosomes, ribosomes, and polyribosomes were fractionated according to the manufacturer's protocol (Teledyne ISCO). A Teledyne ISCO Foxy R1 density gradient fractionator was used to fractionate and analyze gradients with continuous monitoring at $\mathrm{OD}_{254}$.

For assaying the $r p f 2 \Delta 255-344$ mutant strain and the corresponding wild-type control strain, cells were grown in YEPGal overnight and those starter cultures were used to inoculate $250 \mathrm{~mL}$ cultures in YEPGlu, to deplete endogenous Rpf2. Cells were prepared for sucrose gradient fractionation as described above.

Assaying pre-rRNA processing. Northern hybridization and primer extension were used to assay steady-state levels of pre-rRNAs and rRNAs. Strains were grown in either galactose- or glucose-containing medium and $10-20 \mathrm{~mL}$ of cultures were collected and used for RNA extractions. Cells were resuspended in $300 \mu \mathrm{L}$ of RNA buffer $(500 \mathrm{mM} \mathrm{NaCl}, 200 \mathrm{mM}$ Tris-HCl pH 7.5, $10 \mathrm{mM}$ EDTA) and $300 \mu \mathrm{L}$ of PCI (phenol: chloroform : iso amyl alcohol $50: 49: 1$ ) was added to each sample. Cells were vortexed eight times for $30 \mathrm{~s}$ in the presence of glass beads $(0.5 \mathrm{~mm}$ diameter, Biospec Products) and incubated on ice in between vortexing. Samples were centrifuged and the top layers were collected. RNA was precipitated with $100 \%$ ethanol overnight at $-20^{\circ} \mathrm{C}$. Samples were washed with $70 \%$ ethanol and resuspended in water. A Nano Drop 2000C spectrophotometer (Thermo Scientific) was used to quantify amounts of total RNA extracted from cells and $5 \mu \mathrm{g}$ of RNA were used per sample for each reaction.

For northern hybridization, samples were mixed with two volumes of sample buffer ( $8 \%$ formaldehyde, 1.3× MOPS buffer (1 mM sodium EDTA, $20 \mathrm{mM} \mathrm{3-}(\mathrm{N}$ - morpholino propane sulfonic acid pH 7.5, $8 \mathrm{mM}$ sodium acetate)), $65 \%$ formamide, $0.02 \%$ xylene cyanol, and $0.1 \%$ bromophenol blue), and subjected to electrophoresis on a $1.2 \%$ agarose gel for $5.5 \mathrm{~h}$ at $150 \mathrm{~V}$, with continuous recirculation of $1 \times$ MOPS buffer. RNA was transferred by capillary action from the gel to a Zeta-Probe blotting membrane (Biorad). Oligonucleotides were end-labeled using $32 \mathrm{P} \gamma$-ATP and polynucleotide kinase, and hybridized with the membrane at $47-48^{\circ} \mathrm{C}$ overnight in hybridization buffer $(1 \%$ bovine serine albumin, $0.5 \mathrm{mM}$ $\mathrm{Na}_{2} \mathrm{HPO}_{4} \cdot 7 \mathrm{H}_{2} \mathrm{O} / \mathrm{H}_{3} \mathrm{PO}_{4} \mathrm{pH} 7.2,1 \mathrm{mM}$ EDTA pH 8 , and $5 \%$ SDS). Blots were washed three times in wash buffer $(3 \mathrm{M} \mathrm{NaCl}, 300 \mathrm{mM} \mathrm{Na}$ citrate $\mathrm{pH} 7.0$, and $0.2 \%$ SDS) at room temperature and were exposed to X-ray film for one to several days, depending on the abundance of the RNA species detected.

For northern hybridization of small molecular weight RNAs (7S, 5.8S, and 5S), RNA samples were mixed with an equal volume of sample buffer $(0.1 \times$ TBE buffer, $10 \mathrm{M}$ urea, $0.1 \%$ xylene cyanol, $0.1 \%$ bromophenol blue) and subjected to electrophoresis on a $5 \%$ acrylamide/ $7 \mathrm{M}$ urea gel for $4 \mathrm{~h}$ at $120 \mathrm{~mA}$. Following electrophoresis, gels were electroblotted to a Nytran N membrane (GE Healthcare Life Sciences) using a Trans-Blot Plus Cell (Biorad), hybridized with an end-labeled oligonucleotide, washed, and exposed to X-ray film, as described above.

Primer extension assays were performed as follows: radiolabeled oligonucleotide primers complementary to $27 \mathrm{~S}$ pre-rRNA were annealed to RNAs for $10 \mathrm{~min}$ at $46^{\circ} \mathrm{C}$. Reverse transcriptase, RNasin, and deoxynucleoside triphosphates (Promega) were added to the mixture and incubated for $40 \mathrm{~min}$ at $46^{\circ} \mathrm{C}$. RNA was hydrolyzed with $\mathrm{NaOH}$ and EDTA for $30 \mathrm{~min}$ to $1 \mathrm{~h}$ at $55^{\circ} \mathrm{C}$. The reaction was neutralized with $\mathrm{HCl}$ and DNA was precipitated with glycogen, $\mathrm{NH}_{4} \mathrm{OAc}$, and $100 \%$ ethanol. DNA was suspended in DNA dye (95\% formamide, $0.05 \%$ xylene cyanol, $0.05 \%$ bromophenol blue in $20 \mathrm{mM}$ EDTA pH 8.0) and subjected to electrophoresis on a $6 \%$ polyacrylamide-urea sequencing gel. Gels were dried and directly exposed to film for autoradiography for one to several days. 
Oligonucleotide probes or primers are listed in ref. ${ }^{52}$ (Table 4 and Fig. 6).

Fluorescence microscopy. A reporter system that expresses the nucleolar marker mRFP-Nop1 and the L25-enhanced green fluorescent protein (L25-eGFP) (a gift from J. Bassler) was used to visualize a block in nucleolar release and nuclear export of assembling $60 \mathrm{~S}$ ribosomal subunits. Strains expressing truncated Rpf2 protein or lacking Sdal were transformed with the pRS316-RPL25eGFP/mRFPNOP1 plasmid, grown in the appropriate media lacking uracil, and shifted to either $16^{\circ} \mathrm{C}$ for $5 \mathrm{~h}$ ( $r$ pf2 $\left.2255-344\right)$ or to glucose-containing media for $17 \mathrm{~h} \mathrm{(GAL-SDA1).}$ Cells were anchored to MaTek plates using $20-30 \mu \mathrm{L}$ of $1 \mathrm{mg} / \mathrm{mL}$ concanavalin A (ConA, Fisher Scientific). The ConA was dried on plates for $30-45 \mathrm{~min}$ before adding cells. Cell images were obtained by a Zeiss LSM 880 laser scanning confocal microscope at $\times 600$ magnification. Images were acquired using ZEN software (blue edition, by Zeiss) and the images were processed using Fiji for Mac OSX (National Institutes of Health).

Affinity purifications of assembling $60 \mathrm{~S}$ subunits. Ribosome assembly intermediates were affinity-purified from whole-cell extracts with magnetic Dynabeads (Thermo Fisher Scientific), using TAP-tagged AFs Nop7 or Nog2. Cultures $(250 \mathrm{~mL})$ were grown either in galactose- or glucose-containing liquid media to an $\mathrm{OD}_{600}$ of $0.7-0.9$. Cells were collected and resuspended in $3.5 \mathrm{~mL}$ of Lysis Buffer (50 mM Tris- $\mathrm{HCl} \mathrm{pH} 7.5,150 \mathrm{mM} \mathrm{NaCl}, 10 \mathrm{mM} \mathrm{MgCl}, 0.075 \%$ detergent octylphenoxypolyethoxyethanol), and subjected to vortexing with glass beads $(0.5 \mathrm{~mm}$ diameter, Biospec Products) eight times for $30 \mathrm{~s}$, with incubation on ice in between vortexing. Extracts were clarified by centrifugation and bound to IgG-coated Dynabeads at $4{ }^{\circ} \mathrm{C}$ for $1 \mathrm{~h}$. Beads were washed three times with the Lysis buffer and pre-ribosomes were eluted by cleaving the TEV protease site within the TAP-tag, using $1-2 \mu \mathrm{L}$ of TEV Protease (Thermo Fisher Scientific). Proteins were precipitated with $10 \%$ trichloroacetic acid (TCA), resuspended in SDS sample buffer and separated by SDS-PAGE on 4-20\% Tris-Glycine Novex gels (Thermo Fisher Scientific).

Western hybridization analysis. Proteins from whole-cell extracts or from purified pre-ribosomes were separated on SDS-PAGE gels, electroblotted to the Amersham Protran supported $0.45 \mu \mathrm{m}$ NC membrane (GE Healthcare Life Sciences), and assayed by western blot analysis. To conserve antiserum by using a lower volume of blotting buffer and to enable detection of multiple proteins on one blot, nitrocellulose membranes were cut into smaller sections based on the known mobility of the different proteins. As Nog2 co-migrates with IgG on $4-20 \%$ TrisGlycine Novex gels, NuPage 4-12\% Bis-Tris gels (Thermo Fisher Scientific) were used to assay Nog2 protein by western blotting. TAP-tagged proteins were detected using alkaline phosphatase conjugated to IgG (Pierce). HA-tagged proteins were identified with mouse monoclonal antibody 12CA5 (Thermo Fisher Scientific) and Myc-tagged proteins with 9e10 antibody (Sigma-Aldrich). Otherwise, antibodies specific for r-proteins or AFs were used. Alkaline-phosphatase-conjugated antimouse or anti-rabbit secondary antibodies (Promega) were used and colorimetric detection was performed using NBT and BCIP (Promega).

Analysis of pre-60S subunits by iTRAQ. For semi-quantitative mass spectrometry (iTRAQ), pre-ribosomes were purified as described above, with the following modifications. Cell pellets were resuspended in TNM150 buffer $(50 \mathrm{mM}$ Tris-HCl $\mathrm{pH} 7.5,150 \mathrm{mM} \mathrm{NaCl}, 1.5 \mathrm{mM} \mathrm{MgCl}_{2}, 0.1 \% \mathrm{NP}-40$, and $5 \mathrm{mM}$ 2-mercaptoethanol (Sigma-Aldrich)). After incubation of the lysates with IgG-coated Dynabeads for $1 \mathrm{~h}$ at $4{ }^{\circ} \mathrm{C}, \mathrm{NP}-40$ was omitted from the buffer for all later steps. Purified samples were sent to the Penn State Hershey Core Research Facilities for trypsin digestion and 8-plex labeling with iTRAQ reagents 113,114, 115, 116, 117, 118, 119, and 121 (Applied Biosystems). Peptides were separated by two-dimensional (2D) liquid chromatography and parent ions were identified on a Sciex 5600 liquid chromatography mass spectrometer system. Protein Pilot 5.0 was used to obtain iTRAQ ratios as an average of all peptides for each protein. Proteins identified with $>99.9 \%$ confidence were used for further data analysis. Data were normalized to the change in ratio of the Nog2 bait protein.

Purifications of pre-60S subunits for cryo-EM. Pre-ribosomes were purified as described above, with the following modifications: the same protocol as above was used, but the eluates were not precipitated using TCA. Instead, they were concentrated using Amicon-Ultra $0.5 \mathrm{~mL}$ centrifugal filters, Ultracel-100K (Millipore).

Cryo-EM data acquisition. Vitrified specimens were prepared by adding $4 \mu \mathrm{l}$ samples of $r p f 2 \triangle 255-344$ or GAL-SDA1 particles at a concentration of $\sim 150 \mathrm{nM}$ to a glow-discharged holey carbon grid (Quantifoil R2/2) covered with a freshly made thin carbon film. Grids were blotted for $1 \mathrm{~s}$ and plunge-frozen into liquid ethane using an FEI Vitrobot Mark IV $\left(4{ }^{\circ} \mathrm{C}\right.$ and $100 \%$ humidity). The cryo-grids were initially screened at a nominal magnification of $\times 92,000$ in an FEI Talos Arctica microscope, operated with an acceleration voltage of $200 \mathrm{kV}$. Good-quality grids were transferred to an FEI Titan Krios electron microscope that was operating at $300 \mathrm{kV}$ and images were recorded using a K2 Summit direct electron detector (Gatan) in counting mode at a nominal magnification of $\times 130,000$, corresponding to a pixel size of $1.057 \AA$ at the object scale and with the defocus varying from -1.0 to $-2.0 \mu \mathrm{m}$. Coma-free alignment was manually optimized and parallel illumination was verified before data collection. All micrographs obtained with the K2 camera were collected semi-automatically by SerialEM ${ }^{53}$, under low-dose conditions. Each micrograph was dose fractionated to 32 frames with a dose rate of $\sim 8.0$ counts per physical pixel per second for a total exposure time of $8 \mathrm{~s}$.

Cryo-EM data processing. Original image stacks were summed and corrected for drift and beam-induced motion at the micrograph level using the MotionCor2 program $^{54}$. The SPIDER ${ }^{55}$ and EMAN2 programs ${ }^{56}$ were used for micrograph screening and automatic particle picking, respectively. The contrast transfer function parameters of each micrograph were estimated by Gctf ${ }^{57}$. All 2D and 3D classification and refinement were performed with RELION2.1 ${ }^{58}$. For the $r p f 2 \Delta 255$ 355 mutant sample, a total of 3189 micrographs were collected and 272,879 particles were picked for cascade $2 \mathrm{D}$ and $3 \mathrm{D}$ classification with a binning factor of 4 . About $50 \%$ of particles were removed during 2 rounds of $2 \mathrm{D}$ classification and 143,868 particles were subjected to two rounds of 3D classification (Supplementary Fig. 5). After the final round of 3D classification, a total of 12,800 particles were subjected to high-resolution refinement (without binning), generating a density map at an overall resolution of $6.0 \AA$ (C1 class) (gold-standard FSC 0.143 criteria). The B2 and B6 classes of the final round of 3D classification were combined for further high-resolution structural refinement, resulting in a $5.9 \AA$ map (C2). A total of 33,582 particles from B3, B4, and B5 classes were applied for high-resolution refinement (without binning), resulting in a $3.9 \AA$ map (C3). A total of 53,177 particles from C2 and C3 classes were applied for high-resolution refinement with a soft mask (without binning), resulting in a $3.2 \AA$ map (C4).

For pre-ribosomes from the GAL-SDA1 strain, a total of 1224 micrographs were collected and 169,407 particles were picked for cascade 2D and 3D classification with a binning factor of two. About $50 \%$ of particles were removed during two rounds of 2D classification and 73,883 particles were subjected to one round of 3D classification (Supplementary Fig. 7). After the final round of 3D classification, a total of 50,552 particles from the D1 and D3 classes were applied for highresolution refinement (without binning), resulting in a $5.9 \AA$ map (E1). A total of 14,186 particles from D2 and D4 classes were applied for high-resolution refinement (without binning), resulting in a $4.8 \AA ̊$ map (E2).

C4 model building and refinement. An atomic model of the wild-type Nog2-TAP State 1 (PDB accession number 3 JCT $)^{27}$ was used as the initial template for modeling. The models of the rRNAs (25S, 5.8S, 5S, ITS2 RNA) were docked into the density map manually using UCSF Chimera ${ }^{59}$. For r-protein and AF modeling, structures of individual proteins were separately fitted into its density by rigid-body docking. After the initial fitting, the entire chains of rRNAs and proteins were manually checked and adjusted with COOT ${ }^{60}$. The atomic model of $\mathrm{C} 4$ of the $r p f 2 \Delta 255-344$ particles was further refined against the density map first by realspace refinement (phenix.real_space_refine) ${ }^{61}$ in PHENIX ${ }^{62}$, with secondary structure restraints, geometry restraints, and RNA-specific restraints applied. After refinement, alternating rounds of manual model adjustment using COOT and model refinement using PHENIX were applied.

High-copy suppression screen. High-copy plasmids containing the RPF2, RRS1, NOG1, SDA1, RSA4, or CGR1 genes (Yeast genomic tiling collection, Open Biosystems) were transformed into the rpf2 $\triangle 255-344$ mutant strain. Serial dilutions $(1: 10$ to $1: 10,000)$ were spotted onto YEPGlu solid medium and incubated at 30 and $13^{\circ} \mathrm{C}$.

Statistics and reproducibility. Experiments shown in Fig. 1a, b were performed twice. The experiment shown in Fig. 2a was performed three times with yeast expressing the genomic $r p f 2 \Delta 255-344$ mutant strain, and twice with yeast expressing the plasmid-borne mutant allele. The experiment shown in Fig. $2 \mathrm{~b}$ was performed twice with yeast expressing the genomic rpf2 $2255-344$ mutant strain, and once with yeast expressing the plasmid-borne mutant allele. The experiment shown in Fig. $2 \mathrm{c}$ was performed twice with yeast expressing the genomic $r p f 2 \Delta 255-344$ mutant strain, and twice with yeast expressing the plasmid-borne mutant allele. The experiment shown in Supplementary Fig. 4a was performed one time, but those results were backed up by a growth defect observed in the RPF2-TAP strain. The experiment shown in Supplementary Fig. 4e was performed three times. Experiments shown in Supplementary Fig. $4 \mathrm{f}$, g were performed twice. The experiment shown in Supplementary Fig. 5a was performed twice and an average of 150 cells were visualized to confirm the defect. Areas on the micrograph showing the highest numbers of cells were chosen for the publication. Experiments shown in Supplementary Fig. 5b, c were performed twice. Experiments shown in Supplementary Fig. 7a-c, e-g were performed twice. The experiment shown in Supplementary Fig. $7 \mathrm{~d}$ was performed twice, and an average of 150 cells were visualized in order to confirm the defect. Areas on the micrograph showing the highest numbers of cells were chosen for the publication. The experiment shown in Supplementary Fig. $7 \mathrm{~h}$ was performed twice.

PyMOL images. PyMOL images of structures of $60 \mathrm{~S}$ subunit assembly intermediates were generated using PDB 6ELZ [https://doi.org/10.2210/pdb6ELZ/pdb], 
5T62 [https://doi.org/10.2210/pdb5T62/pdb], 3JCT [https://www.wwpdb.org/pdb? id=pdb_00003jct], 5APN [https://doi.org/10.2210/pdb5APN/pdb], and 5H4P [https://doi.org/10.2210/pdb5H4P/pdb] $]^{9,19,27,44,46}$. Pymol images of the structure of yeast mature 60 S ribosomal subunits were generated using PDB file 4V88 [https://doi.org/10.2210/pdb4v88/pdb] ${ }^{7}$.

Reporting summary. Further information on research design is available in the Nature Research Reporting Summary linked to this article.

\section{Data availability}

The data that support this work are available from the corresponding authors upon reasonable request. The cryo-EM density maps of C1, C2, C3, C4 classes of $r p f 2 \triangle 255-344$ mutant particles and E1, E2 classes of Sda1-depleted mutant particles have been deposited in the Electron Microscopy Data Bank under the following accession numbers: EMD-30110 [https://www.ebi.ac.uk/pdbe/entry/emdb/EMD-30110], EMD-30111 [https://www.ebi.ac.uk/pdbe/entry/emdb/EMD-30111], EMD-30112 [https://www.ebi.ac.uk/pdbe/entry/emdb/EMD-30112], EMD-30108 [https://www.ebi.ac.uk/pdbe/entry/emdb/EMD-30108], EMD-30113 [https://www.ebi.ac.uk/pdbe/entry/emdb/EMD-30113], and EMD-30109 [https://www.ebi.ac.uk/pdbe/entry/emdb/EMD-30109], respectively. The atomic model of the C4 class of the $r p f 2 \triangle 255-344$ has been deposited in the Protein Data Bank (PDB) under accession number 6M62 [https://doi.org/10.2210/ pdb6M62/pdb]. Source data are provided with this paper.

Received: 12 November 2019; Accepted: 29 June 2020;

Published online: 27 July 2020

\section{References}

1. Woolford, J. L. Jr \& Baserga, S. J. Ribosome biogenesis in the yeast Saccharomyces cerevisiae. Genetics 195, 643-681 (2013).

2. de la Cruz, J., Karbstein, K. \& Woolford, J. L. Jr. Functions of ribosomal proteins in assembly of eukaryotic ribosomes in vivo. Annu. Rev. Biochem. 84, 93-129 (2015).

3. Nerurkar, P. et al. Eukaryotic ribosome assembly and nuclear export. Int. Rev. Cell Mol. Biol. 319, 107-140 (2015).

4. Kressler, D., Hurt, E. \& Baßler, J. A puzzle of life: crafting ribosomal subunits. Trends Biochem. Sci. 42, 640-654 (2017).

5. Peña, C., Hurt, E. \& Panse, V. G. Eukaryotic ribosome assembly, transport and quality control. Nat. Struct. Mol. Biol. 24, 689-699 (2017).

6. Klinge, S. \& Woolford, J. L. Jr. Ribosome assembly coming into focus. Nat. Rev. Mol. Cell. Biol. 20, 116-131 (2019).

7. Ben-Shem, A. et al. The structure of the eukaryotic ribosome at $3.0 \AA$ resolution. Science 334, 1524-1529 (2011).

8. Gamalinda, M. et al. A hierarchical model for assembly of eukaryotic $60 \mathrm{~S}$ ribosomal subunit domains. Genes Dev. 28, 198-210 (2014).

9. Kater, L. et al. Visualizing the assembly pathway of nucleolar pre-60s ribosomes. Cell 171, 1599-1610 (2017).

10. Sanghai, Z. A. et al. Modular assembly of the nucleolar large subunit processome. Nature 556, 126-129 (2018).

11. Zhou, D. et al. Cryo-EM structure of an early precursor of large ribosomal subunit reveals a half-assembled intermediate. Protein Cell 10, 120-130 (2018).

12. Venema, J. \& Tollervey, D. Ribosome synthesis in Saccharomyces cerevisiae. Annu. Rev. Genet. 33, 261-311 (1999).

13. Ciganda, M. \& Williams, N. Eukaryotic 5S rRNA biogenesis. WIREsRNA 2, 523-533 (2011).

14. Kressler, D. et al. Synchronizing nuclear import of ribosomal proteins with ribosome assembly. Science 338, 666-671 (2012).

15. Calviño, F. R. et al. Symportin 1 chaperones 5 S RNP assembly during ribosome biogenesis by occupying an essential rRNA-binding site. Nat. Commun. https://doi.org/10.1038/ncomms7510 (2015).

16. Donati, G., Peddigari, S., Carol, A., Mercer, C. A. \& Thomas, G. 5S ribosomal RNA is an essential component of a nascent ribosomal precursor complex that regulates the Hdm2-p53 checkpoint. Cell Rep. 4, 87-98 (2013).

17. Zheng, J. et al. Structure of human MDM2 complexed with RPL11 reveals the molecular basis of p53 activation. Genes Dev. 29, 1524-1534 (2015).

18. Nicholas, E. et al. Involvement of human ribosomal proteins in nucleolar structure and p53-dependent nucleolar stress. Nat. Commun. https://doi.org/ 10.1038/ncomms11390 (2016).

19. Barrio-Garcia, C. et al. Architecture of the Rixl-Real checkpoint machinery during pre-60S-ribosome remodeling. Nat. Struct. Mol. Biol. 23, 37-44 (2016).

20. Zhang, J. et al. Assembly factors Rpf2 and Rrs1 recruit $5 \mathrm{~S}$ rRNA and ribosomal proteins rpL5 and rpL11 into nascent ribosomes. Genes Dev. 21, 2580-2592 (2007).
21. Asano, N. et al. Structural and functional analysis of the Rpf2-Rrs1 complex in ribosome biogenesis. Nucleic Acids Res. 43, 4746-4757 (2015).

22. Kharde, S., Calviño, F. C., Gumiero, A., Wild, K. \& Sinning, I. The structure of Rpf2-Rrs1 explains its role in ribosome biogenesis. Nucleic Acids Res. 43, 7083-7095 (2015).

23. Madru, C. et al. Chaperoning 5S RNA assembly. Genes Dev. 29, 1432-1446 (2016).

24. Chen, W., Xie, Z., Yang, F. \& Ye, K. Stepwise assembly of the earliest precursors of large ribosomal subunits in yeast. Nucleic Acids Res. 45, 6837-6847 (2017)

25. Chaker-Margot, M. \& Klinge, S. Assembly and early maturation of large subunit precursors. RNA 25, 465-471 (2019).

26. Leidig, C. et al. $60 \mathrm{~S}$ ribosome biogenesis requires rotation of the $5 \mathrm{~S}$ ribonucleoprotein particle. Nat. Commun. https://doi.org/10.1038/ ncomms4491 (2014).

27. $\mathrm{Wu}, \mathrm{S}$. et al. Diverse roles of assembly factors revealed by structures of late nuclear pre-60S ribosomes. Nature 534, 133-137 (2016).

28. Pöll, G. et al. rRNA maturation in yeast cells depleted of large ribosomal subunit proteins. PLoS ONE. https://doi.org/10.1371/journal.pone.0008249 (2009).

29. Thoms, M. et al. The exosome is recruited to RNA substrates through specific adaptor proteins. Cell 162, 1029-1038 (2015).

30. Wu, S., Tan, D., Woolford, J. L. Jr., Dong, M.-Q. \& Gao, N. Atomic modeling of the ITS2 ribosome assembly subcomplex from cryo-EM together with mass spectrometry identified protein-protein crosslinks. Protein Sci. 26, 103-112 (2016).

31. Baßler, J. et al. The AAA-ATPase Real drives removal of biogenesis factors during multiple stages of 60S ribosome assembly. Mol. Cell 38, 712-721 (2010).

32. Matsuo, Y. et al. Coupled GTPase and remodelling ATPase activities form a checkpoint for ribosome export. Nature 505, 112-116 (2014).

33. Zhang, X. et al. Structural insights into the function of a unique tandem GTPase EngA in bacterial ribosome assembly. Nucleic Acids Res. 42, 430-13,439 (2014).

34. Ali, I. K., Lancaster, L., Feinberg, J., Joseph, S. \& Noller, H. F. Deletion of a conserved, central ribosomal intersubunit RNA bridge. Mol. Cell 23, 865-874 (2006).

35. Borovinskaya, M. A. et al. Structural basis for aminoglycoside inhibition of bacterial ribosome recycling. Nat. Struct. Mol. Biol. 14, 727-732 (2007).

36. Wang, L. et al. Allosteric control of the ribosome by small-molecule antibiotics. Nat. Struct. Mol. Biol. 19, 957-963 (2012).

37. Greber, B. J. Mechanistic insight into eukaryotic 60 S ribosomal subunit biogenesis by cryo-electron microscopy. RNA 22, 1643-1662 (2016).

38. Cepeda, L. P. et al. The ribosome assembly factor Nop53 controls association of the RNA exosome with pre-60S particles in yeast. J. Biol. Chem. 294, 19365-19380 (2019).

39. Nikolay, R. et al. Structural visualization of the formation and activation of the 50S ribosomal subunit during in vitro reconstitution. Mol. Cell 70, 881-893 (2018).

40. Dez, C., Houseley, J. \& Tollervey, D. Surveillance of nuclear-restricted preribosomes within a subnucleolar region of Saccharomyces cerevisiae. EMBO J. 25, 1534-1546 (2006).

41. Thoms, M. et al. Suppressor mutations in Rpf2-Rrs1 or Rpl5 bypass the Cgr1 function for pre-ribosomal 5S RNP-rotation. Nat. Commun. https://doi.org/ 10.1038/s41467-018-06660-w (2018).

42. Manikas, R. G., Thomson, E., Thoms, M. \& Hurt, E. The $\mathrm{K}^{+}$dependent GTPase Nug1 is implicated in the association of the helicase Dbp10 to the immature peptidyl transferase centre during ribosome maturation. Nucleic Acids Res. 44, 1800-1812 (2016).

43. Bradatsch, B. et al. Structure of the pre-60S ribosomal subunit with nuclear export factor Arx1 bound at the exit tunnel. Nat. Struct. Mol. Biol. 19, 1234-1241 (2012)

44. Malyutin, A. G., Musalgaonkar, S., Patchett, S., Frank, J. \& Johnson, A. W. Nmd3 is a structural mimic of eIF5A, and activates the cpGTPase Lsg1 during $60 \mathrm{~S}$ ribosome biogenesis. EMBO J. 36, 854-868 (2017).

45. Ma, C. et al. Structural snapshot of cytoplasmic pre-60S ribosomal particles bound by Nmd3, Lsg1, Tif6 and Reh1. Nat. Struct. Mol. Biol. 24, 214-220 (2017).

46. Vázquez-Laslop, N. \& Mankin, A. S. How macrolide antibiotics work. Trends Biochemical Sci. 43, 668-684 (2018).

47. Gasse, L., Flemming, D. \& Hurt, E. Coordinated ribosomal ITS2 RNA processing by the Las1 complex integrating endonuclease, polynucleotide kinase, and exonuclease activities. Mol. Cell 60, 808-815 (2015).

48. Rodríguez-Galán, O., García-Gómez, J. J., Kressler, D. \& de la Cruz, J. Immature large ribosomal subunits containing the $7 \mathrm{~S}$ pre-rRNA can engage in translation in Saccharomyces cerevisiae. RNA Biol. 12, 838-846 (2015).

49. Biedka, S. et al. Hierarchical recruitment of ribosomal proteins and assembly factors remodels nucleolar pre-60S ribosomes. J. Cell Biol. 217, 2503-2518 (2018). 
50. Longtine, M. S. et al. Additional modules for versatile and economical PCRbased gene deletion and modification in Saccharomyces cerevisiae. Yeast 14, 953-961 (1998).

51. Rigaut, G. et al. A generic protein purification method for protein complex characterization and proteome exploration. Nat. Biotechnol. 17, 1030-1032 (1999).

52. Horsey, E. W., Jakovljevic, J., Miles, T. D., Harnpicharnchai, P. \& Woolford, J. L. Jr. Role of the yeast Rrp1 protein in the dynamics of pre-ribosome maturation. RNA 10, 813-827 (2004)

53. Mastronarde, D. N. Automated electron microscope tomography using robust prediction of specimen movements. J. Struct. Biol. 152, 36-51 (2005).

54. Zheng, S. Q. et al. MotionCor2: anisotropic correction of beam-induced motion for improved cryo-electron microscopy. Nat. Methods 14, 331-332 (2017).

55. Shaikh, T. R. et al. SPIDER image processing for single-particle reconstruction of biological macromolecules from electron micrographs. Nat. Protoc. 3, 1941-1974 (2008).

56. Tang, G. et al. EMAN2: an extensible image processing suite for electron microscopy. J. Struct. Biol. 157, 38-46 (2007).

57. Zhang, K. Gctf: real-time CTF determination and correction. J. Struct. Biol. 193, 1-12 (2016).

58. Scheres, S. H. RELION: implementation of a Bayesian approach to cryo-EM structure determination. J. Struct. Biol. 180, 519-530 (2012).

59. Pettersen, E. F. et al. UCSF Chimera-a visualization system for exploratory research and analysis. J. Comput. Chem. 25, 1605-1612 (2004).

60. Emsley, P., Lohkamp, B., Scott, W. G. \& Cowtan, K. Features and development of Coot. Acta Crystallogr. D 66, 486-501 (2010).

61. Afonine, P. V. et al. Towards automated crystallographic structure refinement with phenix.refine. Acta Crystallogr. D 68, 352-367 (2012).

62. Adams, P. D. et al. PHENIX: a comprehensive Python-based system for macromolecular structure solution. Acta Crystallogr. D 66, 213-221 (2010).

\section{Acknowledgements}

We thank Amber LaPeruta, Fiona Fitzgerald, Melissa Tosiano, Andy Van Demark, Gordon Rule, and Sebastian Klinge for critical reading of this manuscript, technical assistance, and helpful discussions. We thank the following people for their generous gifts of antibodies: Cosmin Saveanu and Micheline Fromont-Racine (Arx1, Nog2, Rlp24, and Nsa2), Janine Maddock (Nog1), Arlen Johnson (rpL8), Francois Lacroute (rpL1), Doug Kellogg (Sda1), Jesús de la Cruz (Erb1), Sabine Rospert (rpL17, rpL26), Vikram Panse (Bud20, Nug1), and Katja Siegers (L25). We thank Jochem Baßler for providing plasmids containing L25-eGFP and mRFP-Nop1. We also thank the Core Facilities at the School of Life Sciences at Peking University for assistance with negative staining EM, and the cryo-EM platform of Peking University for help with data collection. The computational work was supported by the High-performance Computing Platform of Peking University. This work was supported by National Institutes of Health grant R01GM028301 (to J.L. Woolford, Jr.), the National Natural Science
Foundation of China (NSFC) (31725007 and 31630087 to N.G.), and the Ministry of Science and Technology of China (2016YFA0500700 to N.G.)

\section{Author contributions}

J.M. and J.W. designed the study. J.M. constructed strains and plasmids, and assayed effects of mutations on ribosome assembly. J.M. and B.T. prepared samples for cryo-EM. D.W. carried out fluorescence microscopy. Y.L. and S.W. collected the cryo-EM images and Y.L., S.W., and N.G. processed the data. Y.L. built the models and together with J.M., N.G., and J.W. analyzed the structures. J.M., Y.L., N.G., and J.W. interpreted results and wrote the manuscript.

\section{Competing interests}

The authors declare no competing interests.

\section{Additional information}

Supplementary information is available for this paper at https://doi.org/10.1038/s41467020-17534-5.

Correspondence and requests for materials should be addressed to N.G. or J.L.W.

Peer review information Nature Communications thanks Joaquin Ortega, and the other, anonymous, reviewer(s) for their contribution to the peer review of this work. Peer reviewer reports are available.

Reprints and permission information is available at http://www.nature.com/reprints

Publisher's note Springer Nature remains neutral with regard to jurisdictional claims in published maps and institutional affiliations.

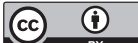

Open Access This article is licensed under a Creative Commons Attribution 4.0 International License, which permits use, sharing, adaptation, distribution and reproduction in any medium or format, as long as you give appropriate credit to the original author(s) and the source, provide a link to the Creative Commons license, and indicate if changes were made. The images or other third party material in this article are included in the article's Creative Commons license, unless indicated otherwise in a credit line to the material. If material is not included in the article's Creative Commons license and your intended use is not permitted by statutory regulation or exceeds the permitted use, you will need to obtain permission directly from the copyright holder. To view a copy of this license, visit http://creativecommons.org/ licenses/by/4.0/.

(C) The Author(s) 2020 